\title{
Aerodynamic Shape Optimization of the CRM Configuration Including Buffet-Onset Conditions
}

\author{
Gaetan K. W. Kenway,* \\ Joaquim R. R. A. Martins ${ }^{\dagger}$ \\ University of Michigan, Department of Aerospace Engineering, Ann Arbor, MI
}

\begin{abstract}
High-fidelity computational modeling and optimization of complex engineering systems has the potential to enable engineers to produce more efficient designs with fewer unforeseen design modifications late in the design process. For transonic wing design, aerodynamic shape optimization has the potential produce high- performance, low-drag designs. Typically, optimizations attempt to improve the performance at a small number of representative operating conditions. However, the performance improvement may be obtained at the expense of reduced robustness or performance in other aspects of the design. In this paper we present a separation-based constraint formulation that can be used to constrain undesirable buffet-onset behavior in an aerodynamic shape optimization. A series of optimizations based on the AIAA Aerodynamic Design Optimization Discussion Group wing-body-tail case are presented to demonstrate the need for buffet-onset constraints, and the effectiveness of the proposed approach. Both single-point and multipoint optimizations without separation constraints are found to be vulnerable to buffeting, while the optimizations with the proposed approach successfully move the buffet boundary to make the designs feasible.
\end{abstract}

\section{Introduction}

Numerical optimization has the potential to be a powerful tool that can complement more traditional design methodologies. Optimization using high-fidelity, physics-based simulations, such as Computational Fluid Dynamics (CFD) and Computational Structural Mechanics (CSM), is especially promising. By capturing the relevant physics of the underlying system, performance improvements predicted by numerical simulations are more likely to be realized in the real world. Effective optimization algorithms, however, invariably exploit any shortcomings in the numerical models and violate important constraints not included in the optimization problem formulation.

One critical aspect of transonic wing design that is not usually considered in aerodynamic shape optimization is the behavior of the design near buffet. As the Mach number or lift coefficient increases, shocks on the wing gradually increase in strength. Interactions of the shocks with the boundary layer cause flow separation, and eventually this leads to unsteady separated flow and oscillation of the shocks. This phenomena leads to an aerodynamically-induced vibration of the airframe. Federal Aviation Administration (FAR) and Civil Aviation Authority (CAA) regulations stipulate that a $1.3 \mathrm{~g}$ margin must exist between a regular operation cruise condition and buffet onset. This corresponds approximately to a $40^{\circ}$ bank angle for a steady-state turn.

To quantify the buffet-onset in aerodynamic shape optimization, we develop a new prediction method based on the amount of separated flow present on the wing. We then use this method to enforce a constraint on the amount of separated flow near the buffet onset boundary, which ensures that the optimized design has a sufficient buffet margin, while simultaneously improving the performance at the design operating conditions.

This paper is organized as follows. First, we outline the key aspects of the computational methods used in this work, then we describe the separation constraint formulation, and how it is used to enforce the buffet onset constraint. Then, we present a sequence of numerical aerodynamic optimization studies based on the AIAA Aerodynamic Design Optimization Discussion Group (ADODG) wing-body-tail case to evaluate the effects of including buffet onset constraints in a transonic wing aerodynamic shape optimization.

\section{Computational Methods}

The aerodynamic analysis code used in this work is part of the MACH (MDO of Aircraft Configurations with High fidelity) framework. This framework was developed to perform aerodynamic and aerostructural design optimization studies of both conventional and unconventional aircraft configurations [1,2].

\footnotetext{
${ }^{*}$ Research Investigator, AIAA Member

${ }^{\dagger}$ Professor, AIAA Associate Fellow
} 


\section{CFD Solver}

The aerodynamic flow solver in MACH is SUMad, which solves the Reynolds-averaged Navier-Stokes (RANS) equations in either steady, unsteady or time spectral modes [3,4]. SUMad employs the finite-volume method on structured, body fitted, multiblock grids. The discretization scheme uses central fluxes with artificial dissipation and the SpalartAllmaras (SA) turbulence model [5]. A matrix dissipation scheme is used except where explicitly noted. A fully coupled Newton-Krylov method is used to solve the mean flow and turbulence equations simultaneously. A discrete adjoint method is implemented using a combination of reverse mode automatic differentiation and analytic methods for the efficient computation of the gradients of functions of interest. More details on the CFD adjoint implementation are provided by Lyu et al. [6].

\section{Geometric Parametrization}

In this work we use an free-form deformation (FFD) volume approach that we have used extensively in the past for aerodynamic $[7,8,9,10,11,12]$ and aerostructural optimization studies [1, 13, 2, 14]. The FFD approach can be visualized as embedding the spatial coordinates defining a geometry inside a flexible volume. The parametric locations $(u, v, w)$ corresponding to the baseline geometry are found using a Newton search algorithm. Once the baseline geometry has been embedded, perturbations made to the FFD volume propagate within the embedded geometry via the evaluation of the nodes at their parametric locations.

\section{Mesh Movement}

The FFD approach previously described applies deformations only to the surface mesh, that is, the part of the volume mesh that lies on the physical surface. A separate operation is then required to propagate surface perturbations to the remainder of the volume mesh. The mesh movement algorithm used in this work is an efficient analytic inverse distance method similar to the one described by Luke et al. [15]. Updating the mesh for a new configuration is fast, typically requiring less than $0.1 \%$ of the time required for a CFD solution. Sensitivities required for the adjoint method are provided from a combination of reverse-mode automatic differentiation and analytic methods.

\section{Optimization Algorithm}

The high computational cost of RANS-based optimization demands an optimization algorithm requiring a low number of function calls. We use SNOPT (sparse nonlinear optimizer) [16] through the Python interface pyOpt [17]. SNOPT is a gradient-based optimizer that implements a sequential quadratic programming method; it is capable of solving largescale nonlinear optimization problems with thousands of constraints and design variables. SNOPT uses an augmented Lagrangian merit function, and the Hessian of the Lagrangian is approximated using a quasi-Newton method. We have successfully employed the SNOPT algorithm to solve a wide variety of aerodynamic and aerostructural optimization problems $[2,14,18,10,7]$.

\section{Buffet Onset Prediction}

In transonic flow, at sufficiently high lift coefficients and Mach numbers, instabilities in the shock-boundary layer interaction cause oscillations in the shock position that result in an unsteady flow field. Pure aerodynamic buffeting can be observed even on an idealized rigid wing. As a real flexible aircraft approaches the buffet boundary, these small aerodynamic oscillations grow in magnitude and induce increasingly large structural vibrations. Eventually, these oscillations may become large enough to cause structural failure or loss of control, especially if the control surfaces are affected.

Current Joint Aviation Requirements (JAR) stipulate that commercial transport aircraft must maintain at least a $1.3 \mathrm{~g}$ margin from the cruise operating condition to buffet onset. This buffet margin may also be considered the maneuvering margin for the aircraft: A $1.3 \mathrm{~g}$ turn corresponds to an approximately $40^{\circ}$ bank angle.

Flight testing certification of new aircraft involves, among many other things, mapping out the flight envelope up to and through the buffet boundary. In practice, for aircraft certification purposes, the buffet boundary is determined when the peak-to-peak acceleration amplitude reaches $n=0.25$ at the pilot's seat. Such a definition is not conducive for inclusion in a conceptual or preliminary design optimization. There are, however, a multitude of other techniques that have been developed over the years to correlate data, typically from wind tunnel experiments, with buffet predictions from a flight testing program. These techniques include correlations with RMS signal strength of wind tunnel model strain gauges, trailing edge pressure divergence, axial force break, pitching moment break, and lift curve break [19]. The last two methods can also be readily applied to the integrated force and moment values obtained from a CFD analysis. One particular way of implementing the lift curve break method is the $\Delta \alpha=0.1$ method. Using this 
method, the "linear" portion of the lift curve is offset toward the right by a value of $0.1^{\circ}$, and the intersection of this line with the actual lift curve is used to estimate the buffet onset point, as illustrated in Fig. 1.

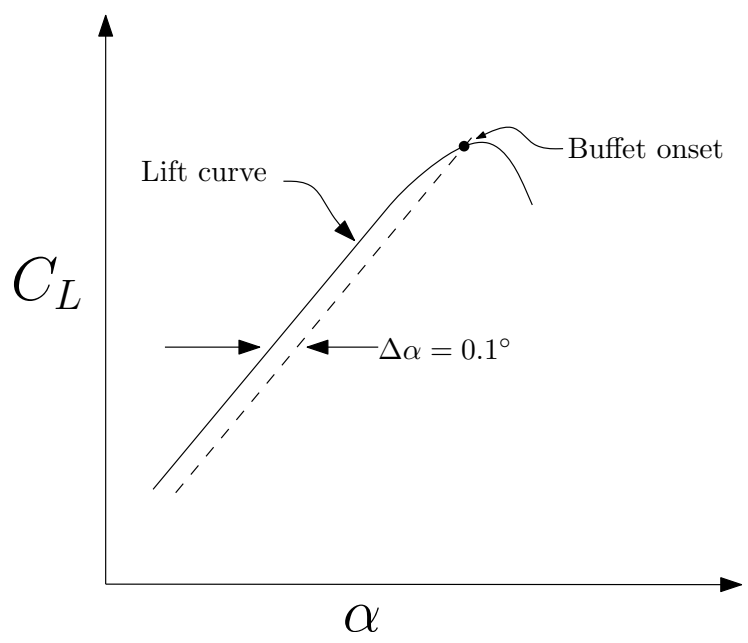

Figure 1: Estimating the buffet boundary with the $\Delta \alpha=0.1$ method.

The physical mechanism that causes the loss of lift and the subsequent lowering of the lift curve slope is the initial appearance of shock-induced flow separation. An example showing the typical progression for a transport aircraft wing at high angles of attack at fixed Mach number is shown in Fig. 2. The red surface in the first row shows the regions where the local surface flow is in the negative streamwise (negative $x$-axis) direction. Alternatively, this is equivalent to the region where the skin friction coefficient, $C_{f_{x}}$, is negative.

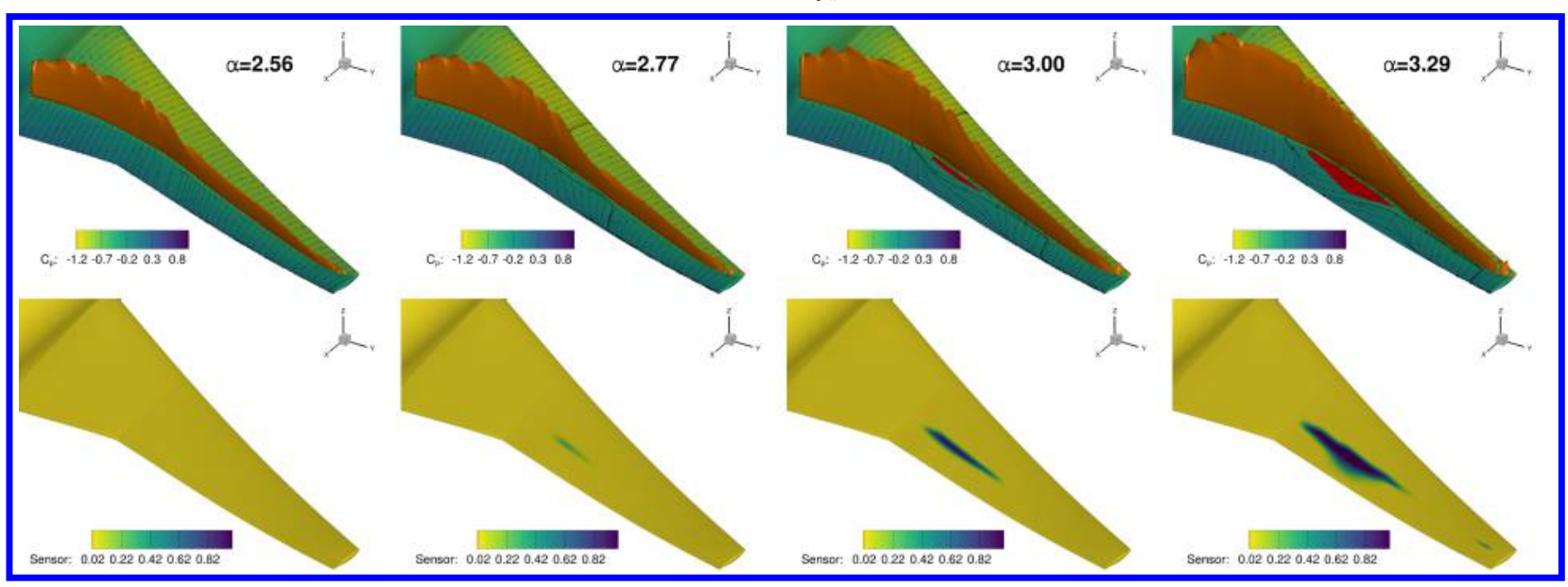

Figure 2: Progression of separated flow for the baseline CRM configuration at $M=0.85$ with increasing angle of attack. The red surface denotes flow in the negative stream-wise direction. The orange isosurface is the Lovely-Haines shock sensor [20]. The last row shows the separation sensor integrand value from Eqn. (1).

We have consistently observed that the appearance of this negative skin friction region correlates with the buffet onset boundary as predicted by the $\Delta \alpha=0.1$ method. This is not surprising, since the appearance of separated flow is the primary cause for the flattening of the lift curve slope. However, the exclusive use of global aerodynamic coefficients-such as $C_{L}$ and $C_{M}$-in determining buffet onset is not strictly necessary for CFD-based shape optimization, as we have the full 3D flow field readily available. To that end, we propose using a separation sensor as a predictor for buffet onset. In its simplest form, the sensor is the integral of the wing surface area where the skin friction is negative. This is equivalent to performing a weighted area integration of the sensor value shown in the bottom row of Fig. 2.

Since our goal is to use this function as a constraint in an optimization, the discontinuity of the function as $V_{x}$ crosses through zero is problematic. To address this issue, we use a smooth Heaviside function to blend the disconti- 
nuity, which yields the following equation for the proposed separation sensor:

$$
\begin{aligned}
\text { Sep } & =\frac{1}{A_{\text {ref }}} \iint_{S} \frac{1.0}{1.0+e^{-2 k(\chi-\lambda)}} \mathrm{d} S, \\
\text { where } \quad \chi & =-\vec{V} \cdot \vec{V}_{\text {freestream }},
\end{aligned}
$$

In the this equation, $k$ and $\lambda$ are free parameters, where $k$ determines the sharpness of the transition, and $\lambda$ is an additional parameter that can be used to shift the smoothing function to the left or right. For our cell-centered solver, the values for $\vec{V}$ are taken from the state variables at the cell center immediately adjacent to the wall, since the velocities at the wall are zero when enforcing the no-slip condition. Both $\vec{V}$ and $\vec{V}_{\text {freestream }}$ are normalized to a unit magnitude, ensuring that the dot product is between -1 and 1 . Since the integral in Eq. (1) amounts to an area, we normalize the sensor value by the aircraft reference area. Smooth Heaviside functions for $\lambda=-0.1,0,1.0$ and $k=10$ are shown in Fig. 3. The last row of Fig. 2 shows the value of $\chi$ on the wing surface at $M=0.85$. The effect of the smooth Heaviside function is to smear out the sensor value around the separated flow region.

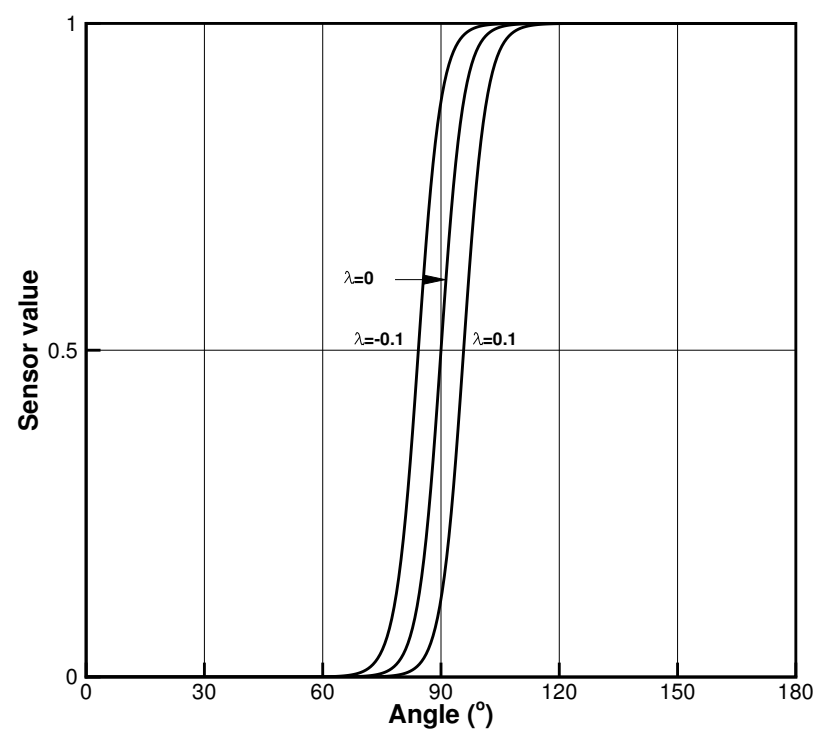

Figure 3: With $k=10$, the majority of the smooth variation occurs over $\pm 15^{\circ}$.

To demonstrate the utility of the separation sensor-based method for predicting buffet onset, we compute the buffet boundary for the baseline CRM configuration [21] at a flight altitude of 37,000 ft over a range of Mach numbers between 0.8 and 0.9 using the $\Delta \alpha=0.1$ method, as well as with the separation sensor criterion. The separation criterion requires the specification of a cutoff value. For this work, we have determined this value by comparing the resulting buffet boundary with the $\Delta \alpha=0.1$ method. The results for Sep $=4.0 \%$ show the best agreement, and the overall shape of the buffet boundary is well matched, as shown Fig. 4.

Some of the discrepancy between the two methods can be explained by analyzing the lift curve slopes for the baseline configuration and one of the optimized configurations presented later in Section V. The "linear" part of the lift curve slope is taken from the first two analysis points at the lowest $C_{L}$ values. Figure 5a graphically shows the $\Delta \alpha=0.1$ method for the baseline configuration. The lift curves are roughly linear, but there is a significant increase in the lift curve slope over the intermediate range of Mach numbers, which flattens the actual lift curve relative to the linear approximation. Therefore, at the higher lift coefficients, the buffet onset is slightly over-predicted when using the $\Delta \alpha=0.1$ method, since a much larger reduction in lift curve slope is necessary to intersect with the linear approximation. For one of the optimized configurations, shown in Case 5b, the effect is much more pronounced. At the higher Mach numbers $(>0.88)$, the slope for the lift curve slope is much closer to the buffet onset location and this effect is not seen. The result is an under-prediction of the buffet onset when compared to the separation based method. The differences in Fig. 4 are consistent with this effect. Additionally, the separation based approach varies more smoothly with respect to Mach number, which is beneficial for gradient-based design optimization.

Figure 6 demonstrates graphically how the separation sensor approach is used to predict the buffet onset boundary. For each $\alpha$ sweep at a fixed Mach number, the intersection of the separation value with the specified cutoff value determines the boundary. With nearly $100 \%$ fully attached flow on the wing upper surface, the sensor value is close 


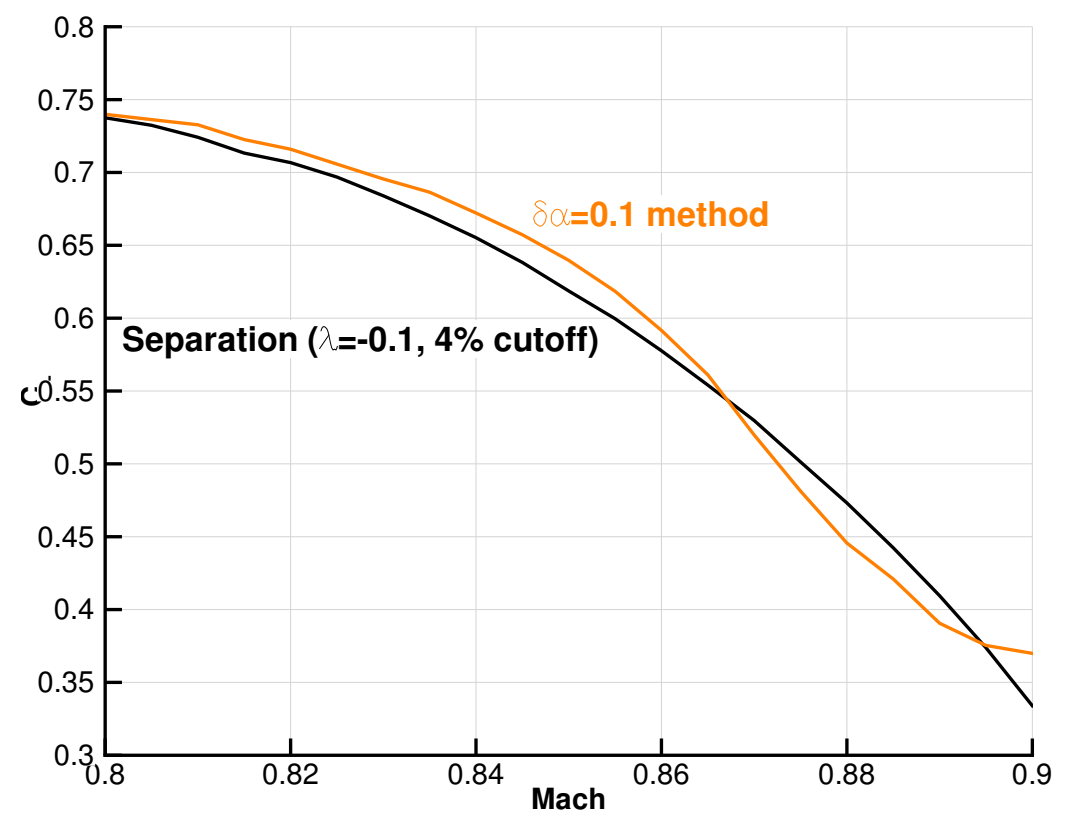

Figure 4: Comparison between the $\Delta \alpha=0.1$ method and the proposed separation sensor method.

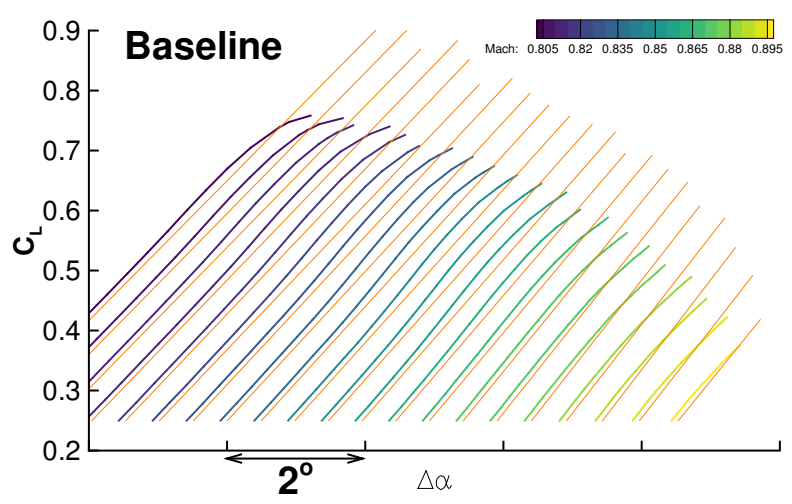

(a) Small variation in lift curve slopes for baseline configuration

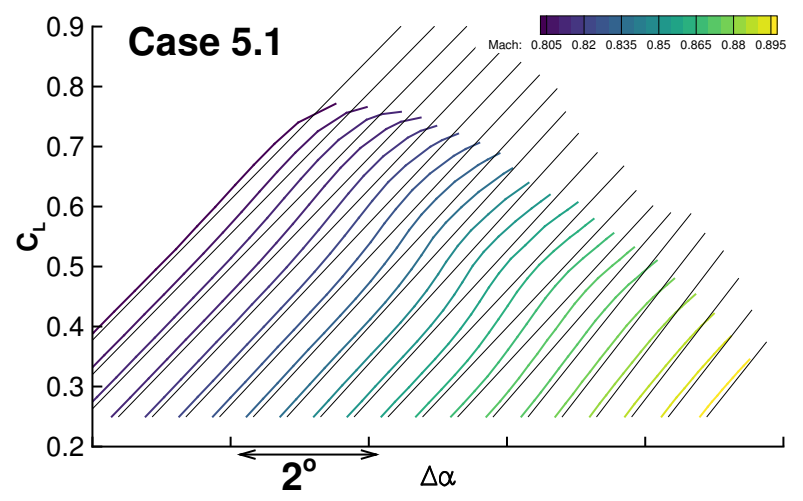

(b) Highly variable lift curve slopes for Case 5.1 optimized configuration, especially over the Mach number range from 0.82 to 0.86 .

Figure 5: Lift curves for baseline and one optimized configuration for Mach 0.8-0.9. Successive lift curves are offset by $0.5^{\circ}$ for visualization purposes.

to zero and then rapidly increases as shock-induced flow separation becomes more severe. The high slope of the separation sensor curve means the predicted $C_{L}$ value for buffet is not particularly sensitive to the selected cutoff value.

The CRM configuration has been extensively tested in various wind tunnels around the world. The results of one such dynamic test are described by Balakrishna and Acheson [22]. In this experiment, the CRM wind tunnel model was instrumented with a wing-root strain gauge. High-speed measurements of the strain gauge allows for the determination of the buffet onset boundary directly by measuring the increase in signal amplitude caused the by the separated flow. Specifically, a buffet coefficient $C_{B}$ is determined based on the area of the wing-root strain spectrum, normalized by the value at zero degrees angle of attack. It is assumed that there is no significant amount of separated flow at zero angle of attack, and that any increase in the value $C_{B}$ as the angle of attack increases can be attributed to 


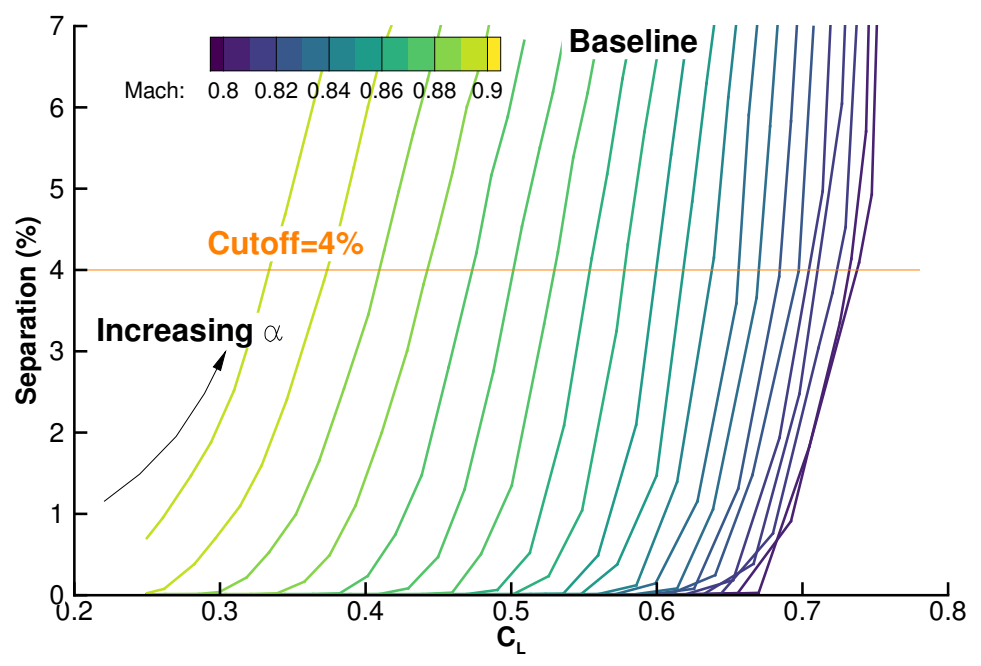

Figure 6: Separation sensor value for $\alpha$ sweeps at a range of Mach numbers. The cutoff value indicates the estimated buffet boundary.

the development of flow separation. Thus, the buffet coefficient is defined as [22],

$$
C_{B}(\alpha)=\frac{\int_{0}^{f_{\max }} S_{\alpha}(f) d f}{\int_{0}^{f_{\max }} S_{0}(f) d f}
$$

Figure 7, reproduced from Figure 4 Balakrishna and Acheson [22], shows the evolution of $C_{B}$ for two Mach numbers: a high subsonic Mach number (0.70) and a transonic Mach number (0.85). We overlaid lines at the $\alpha$ values that yield the separation sensor value of $4 \%$ using our method, with $\lambda=-0.1$. We can see that the separation sensor method correlates well with the increase of $C_{B}$. This indicates we are correctly predicting the buffet onset on the wing.

Figure 8 shows the surface distribution of $C_{p}$ and $\chi$ for the two flow conditions. Due to the differing freestream Mach number, the types of separation and the corresponding separation locations are quite different. For the $M=0.70$ case, there is a separation bubble just aft of the small leading edge sonic region, while for the $M=0.85$ case, the separated flow appears near the mid chord position at the foot of a strong normal shock. While more experimental comparison is required, it appears that the separation sensor does adequately predict buffet onset for both high subsonic and transonic flow conditions.

\section{Aerodynamic Shape Optimization with Buffet Onset}

We now demonstrate the need for considering buffet onset criteria, and the effectiveness of the proposed approach for transonic aerodynamic shape optimization by solving a series of aerodynamic design optimization problems based on the AIAA ADODG Case 5 benchmark [23].

\section{Baseline Geometry}

The baseline geometry defined in the ADODG Case 5 is taken directly from the $4^{\text {th }}$ Drag Prediction Workshop "WingBody-Tail $i_{H}=0$ " configuration [24]. The main reference parameters for this baseline configuration are listed in Table 1.

\section{Computational Grids}

We generated a sequence of four CFD meshes for the CRM wing-body-tail configuration using the meshing software ICEMCFD. There are two grid families: the " 1 series" and the " 0.5 series". Each " 0.5 series" mesh has approximately 2.5 times more cells than the corresponding coarser " 1 series" mesh below it, and has approximately 3.3 times fewer cells than the next finer "1 series" mesh above it. The two coarsest grids-L2 and L1.5-are used for optimization, 


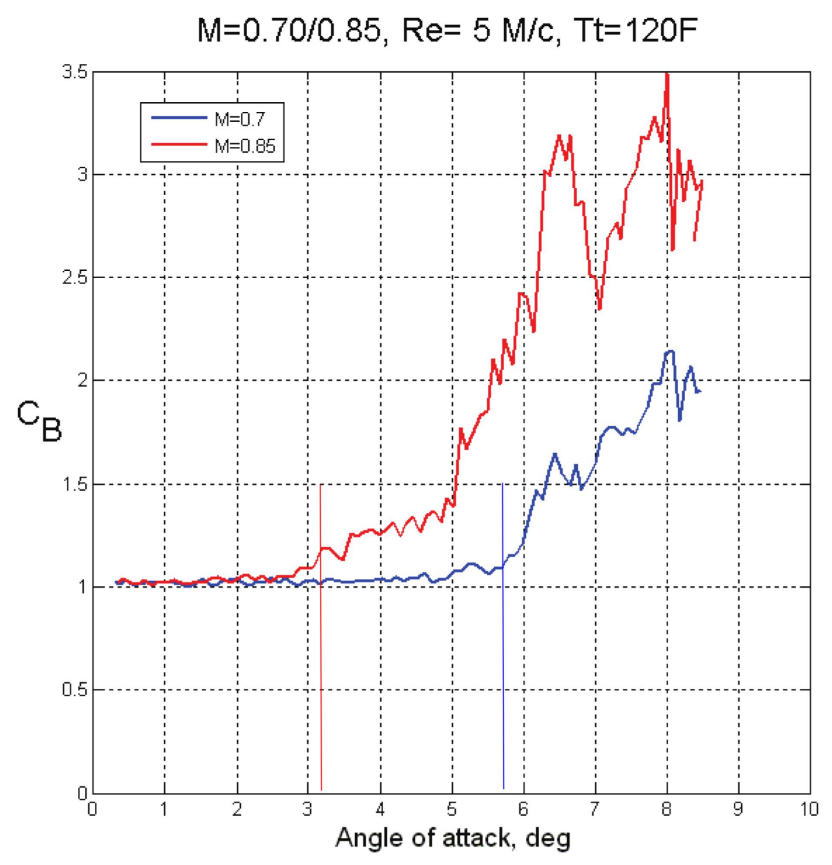

Figure 7: Buffet coefficient $\left(C_{B}\right)$ obtained from wind tunnel data [22]. Vertical lines are the separation sensor predicted buffet onset location.

\begin{tabular}{ll}
\hline Quantity & Value \\
\hline Reference area & $594720.0 \mathrm{in}^{2}$ \\
Reference chord & $275.8 \mathrm{in}$ \\
Moment reference & $(1325.90,0,177.95)$ in \\
Reynolds number $(M=0.85)$ & $43 \times 10^{6}$ \\
\hline
\end{tabular}

Table 1: Reference quantities for CRM full configuration [24].

while the two finest grids- $\mathrm{L} 1$ and L0.5-are used only for post-optimization verification purposes. The mesh characteristics are summarized in Table 2. A comparison of the surface mesh resolution of the four meshes is shown in Fig. 9. Grid convergence studies for the baseline mesh and all optimized configurations are presented in Section 3.

\begin{tabular}{crrcrr}
\hline Mesh level & $\begin{array}{r}\text { Chordwise } \\
\text { Cells }\end{array}$ & $\begin{array}{r}\text { Spanwise } \\
\text { Cells }\end{array}$ & $y_{\max }^{+}$ & $\begin{array}{r}\text { Total } \\
\text { Cells }\end{array}$ & $\begin{array}{r}\text { Baseline } \\
C_{D} \text { (counts) }\end{array}$ \\
\hline L0.5 & 224 & 144 & $\sim 0.5$ & 14233600 & 231.15 \\
L1 & 168 & 108 & $\sim 0.7$ & 5921536 & 234.87 \\
L1.5 & 112 & 72 & $\sim 1.1$ & 1779200 & 249.47 \\
L2 & 84 & 54 & $\sim 1.7$ & 740192 & 269.76 \\
\hline
\end{tabular}

Table 2: Mesh characteristics and trimmed drag coefficient for baseline configuration.

\section{Optimization Problem Statement}

A sequence of seven design optimizations are solved to study the aerodynamic shape optimization of the ADODG full CRM configuration, and to demonstrate the effectiveness of the proposed approach in satisfying buffet requirements. These cases-numbered 5.1 through 5.7-are summarized in Table 3. Only Cases 5.1 and 5.2 are currently specified 


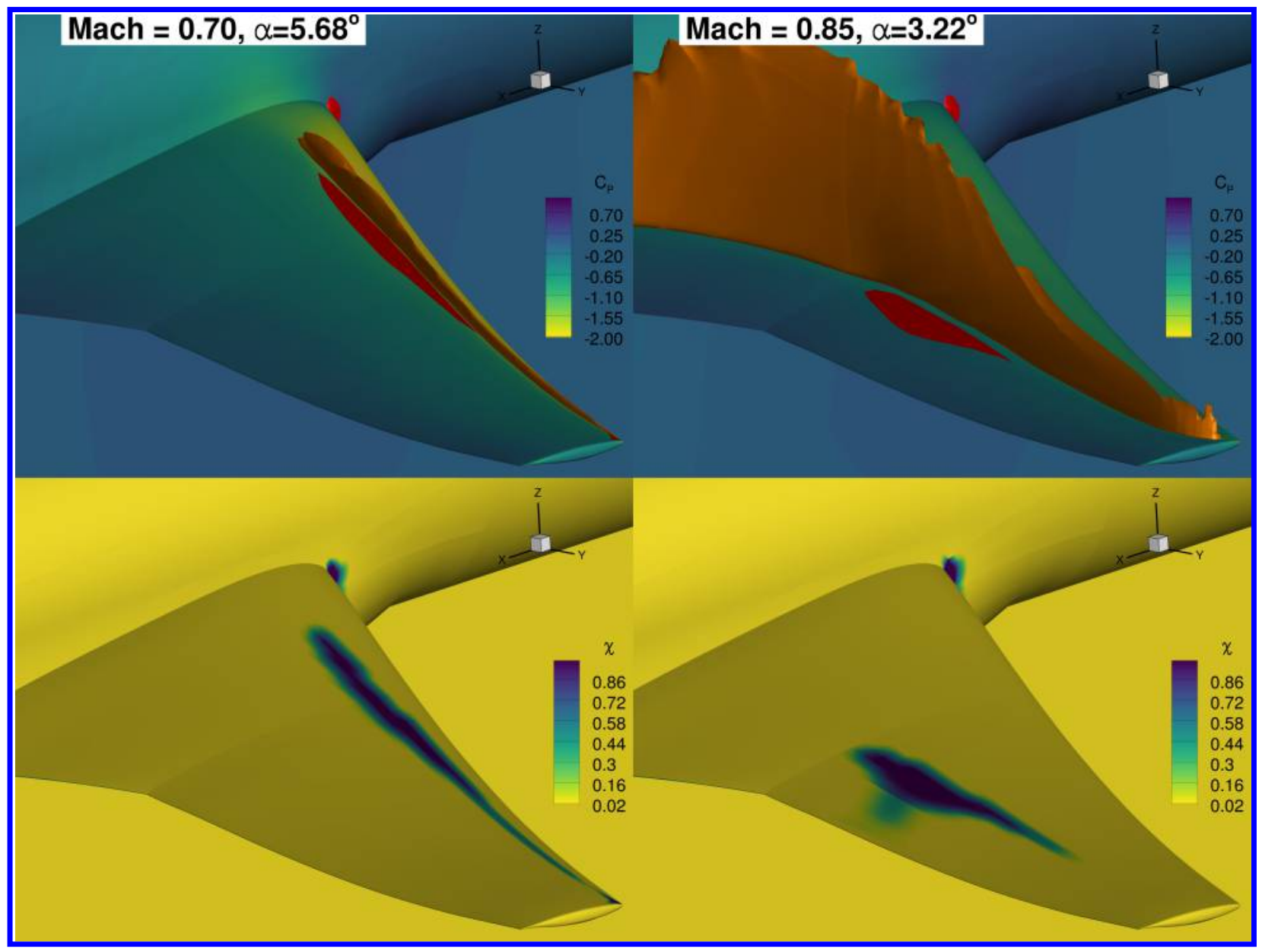

Figure 8: $C_{p}$ and $\chi$ surface distribution for the two experimental buffet onset conditions. In both cases, the separation (red) appears behind the shock (orange).

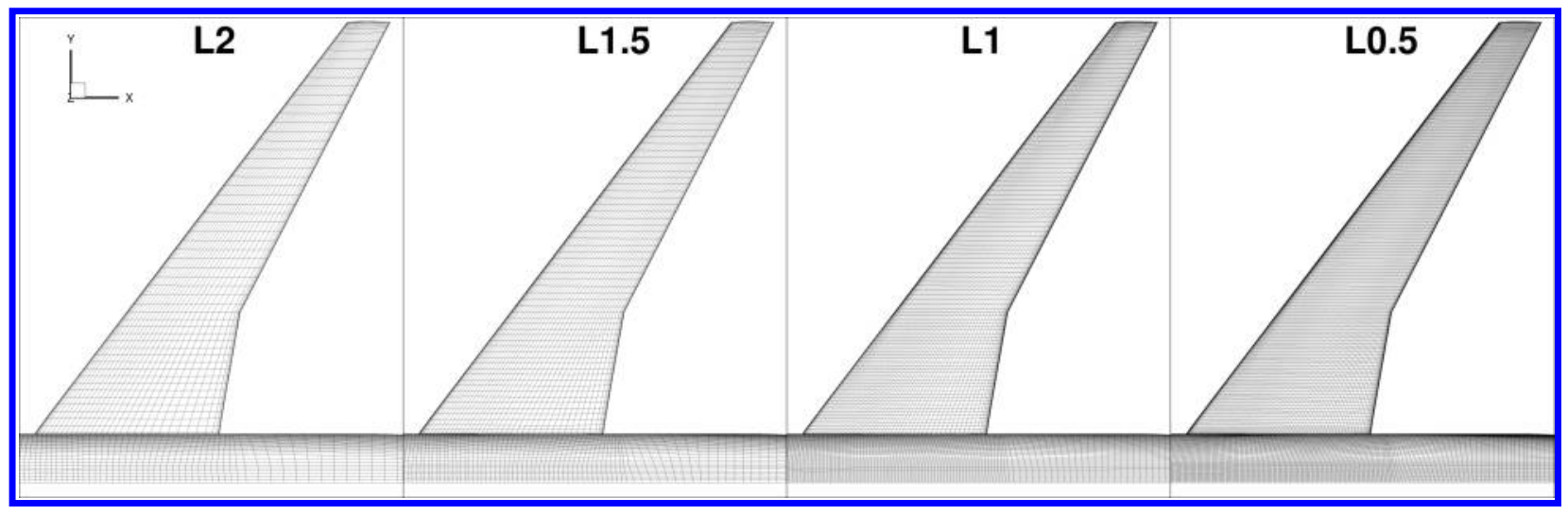

Figure 9: Spatial resolution for each mesh size.

by the ADODG [23]. We added the other cases (Cases 5.3 through 5.7) to further study the effects of including buffet onset conditions on the optimized geometries. The objective of all optimizations (except for Case 5.7, which will be discussed separately) is to reduce the weighted drag coefficient at the $N$ defined operating conditions. The optimization problem statement can be written as: 
minimize $\sum_{i=1}^{N} \mathcal{W}_{i} C_{D_{i}} \quad$ Quantity

with respect to Wing cross sectional shape 240

Wing twist 9

Angle of attack $\left(\alpha_{i}\right) \quad \mathrm{N}$

Tail rotation angle $\left(\eta_{i}\right) \quad \mathrm{N}$

subject to $C_{L_{i}}-C_{L_{i}}^{*}=0.0 \quad \mathrm{~N}$

$C_{M_{y_{i}}}=0.0 \quad \mathrm{~N}$

$t_{j} \geq t_{\text {CRM }} \quad 750$

$\operatorname{Sep}_{i} \leq 0.04 \quad \mathrm{~N}$

Only Cases 5.4, 5.5, and 5.7 use the separation constraint, where the constraint is applied only to the last two operating conditions. In practice, the adjoint for $C_{D}$ is not evaluated for the buffet onset conditions, and conversely the separation sensor adjoint is not evaluated for the normal operating conditions. This results in a total of three adjoint solutions required for each flight condition, which is desirable from a load balancing perspective. The constraints that the wing thickness may not be less than the baseline configuration at any location is particularly restrictive. Essentially, only changes in the wing camber line are available to the optimizer, since it highly desirable in transonic flow to make the wing as thin as possible [25]. The thickness constraints ensure that the wing does not become excessively thin, since this would result in a significant structural weight increase.

The ADODG specification for Case 5 requires the parameterization not to modify the planform, and any shape modification must be made only in the vertical direction. Additionally, twist rotation is permitted for the wing, as well as a solid-body rotation of the horizontal tail for trimming the aircraft. We use the FFD approach described in Section II. The FFD volume and the associated geometric design variables are shown in Fig. 10.

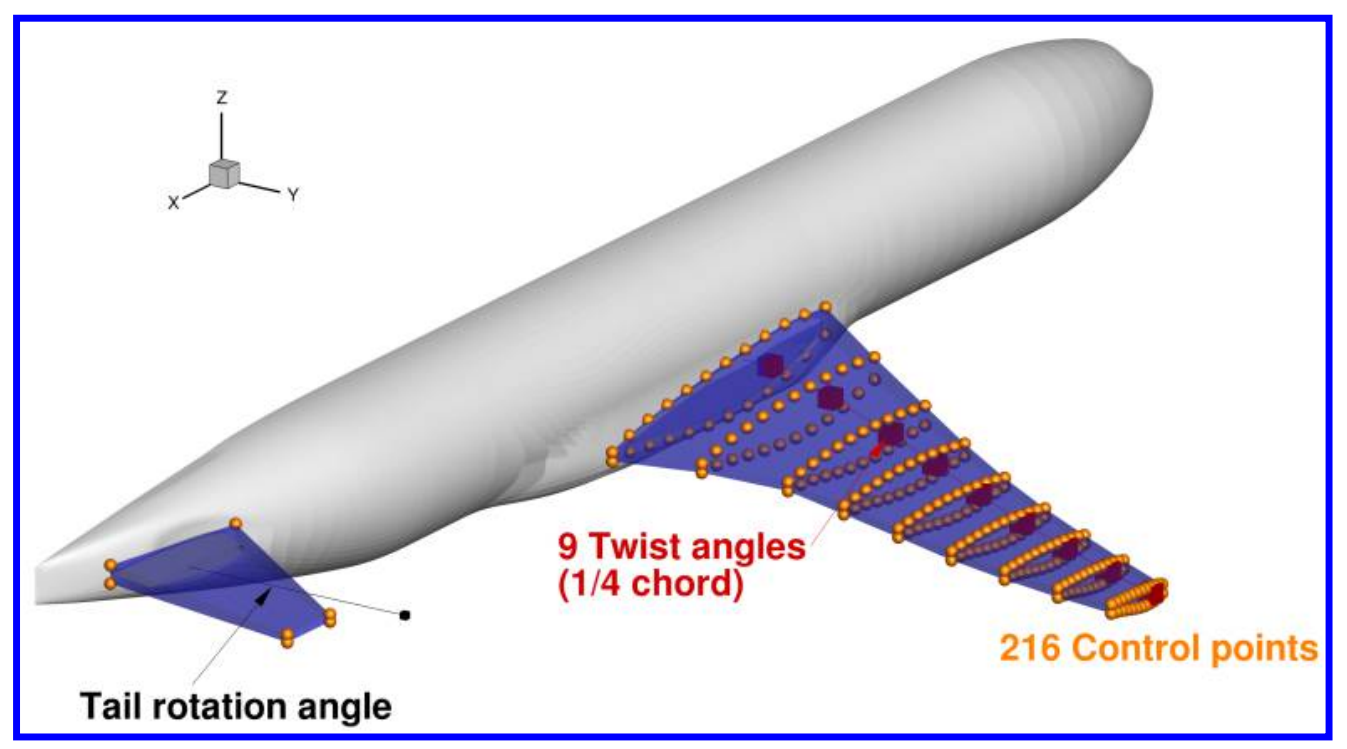

Figure 10: Shape and twist modification are permitted for the wing, and the horizontal tail can rotate.

\section{Results}

\section{Multilevel Approach}

To reduce the overall computational cost of performing the optimizations, we employ the multilevel optimization approach described previously by the authors [10, 25]. Optimizations are first carried out on the coarsest mesh (L2), and the resulting optimum design becomes the starting design for the next finner mesh (L1.5), and so on. In this work, only the first two grid levels are used for optimization. Because of the lower cost of optimizing on the coarse grid, we can afford to do more iterations on this grid. For this approach to be effective, the coarse grid must capture the main characteristics of the flow.

A comparison between the baseline and optimized designs for Case 5.1 is shown in Fig. 11. The first row shows the baseline and optimized designs using the L2 grid. The second row shows the L1 optimization using the L2 optimized shape as the starting point. Color-coded slices of the airfoil shapes and the corresponding $C_{p}$ distributions are shown for four spanwise locations at the bottom of the figure. We see that the coarse optimization (using the L2 
Table 3: Operating conditions for each optimization. Red diamonds denote separation constrained points. Operating conditions for Case 5.7 are determined by the optimization process itself. Zero weight values mean the flight condition is considered only in the constraints.

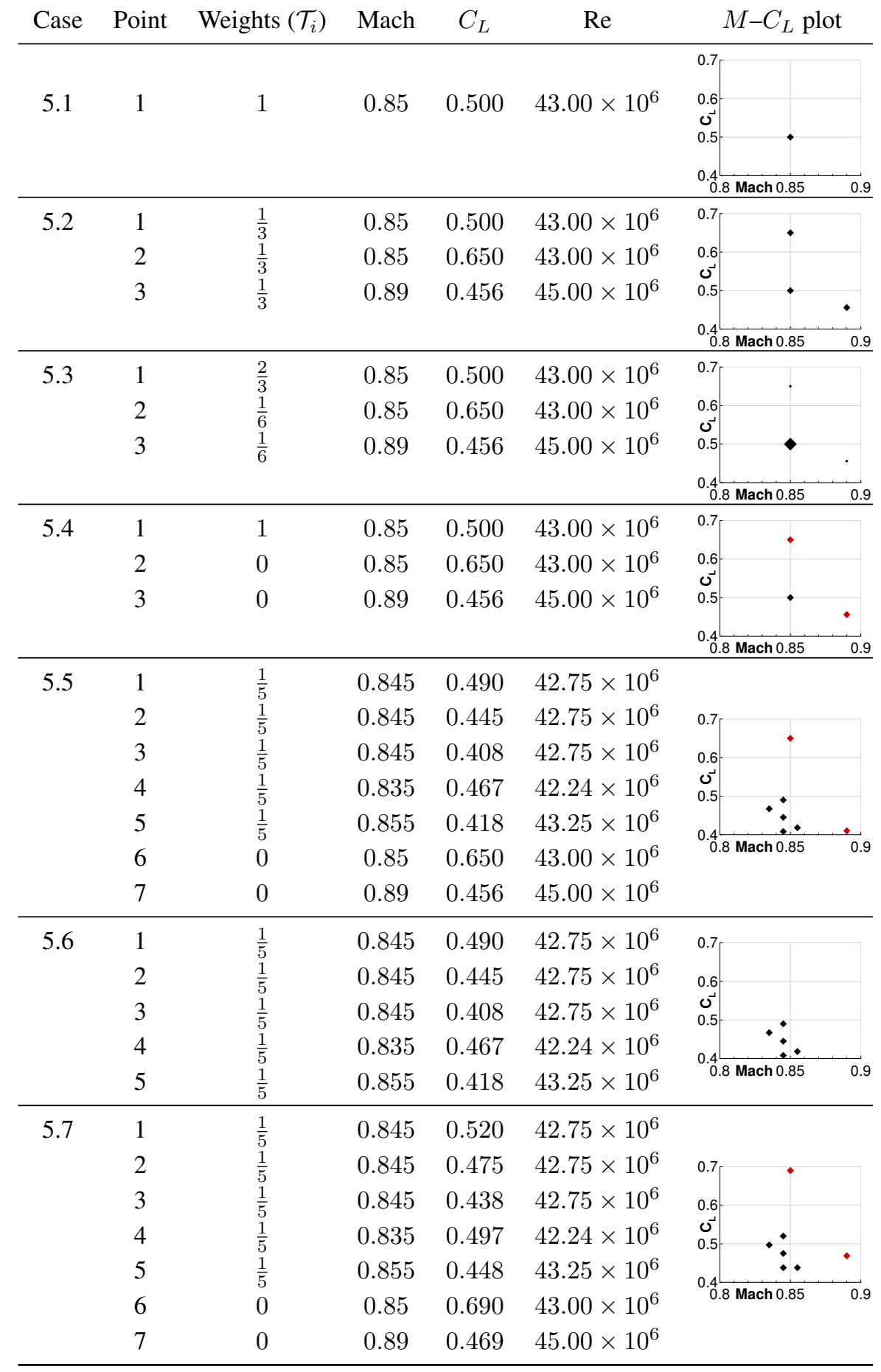

grid) successfully eliminates the shock on the upper wing surface, resulting in parallel isobars. Even without further optimization, almost all of the drag improvement predicted by the coarse grid is realized on the fine grid. Comparing the orange and black lines on the $C_{p}$ distributions, we see that the only significant difference is the appearance of a weak shock on the refined grid. The fine optimization further improves the design, eliminating this shock and lowering the drag even further. This behavior is consistent with our previous results, where we used three uniformly-refined grid levels [10]. We employ this multilevel approach for all the optimizations in this paper. 


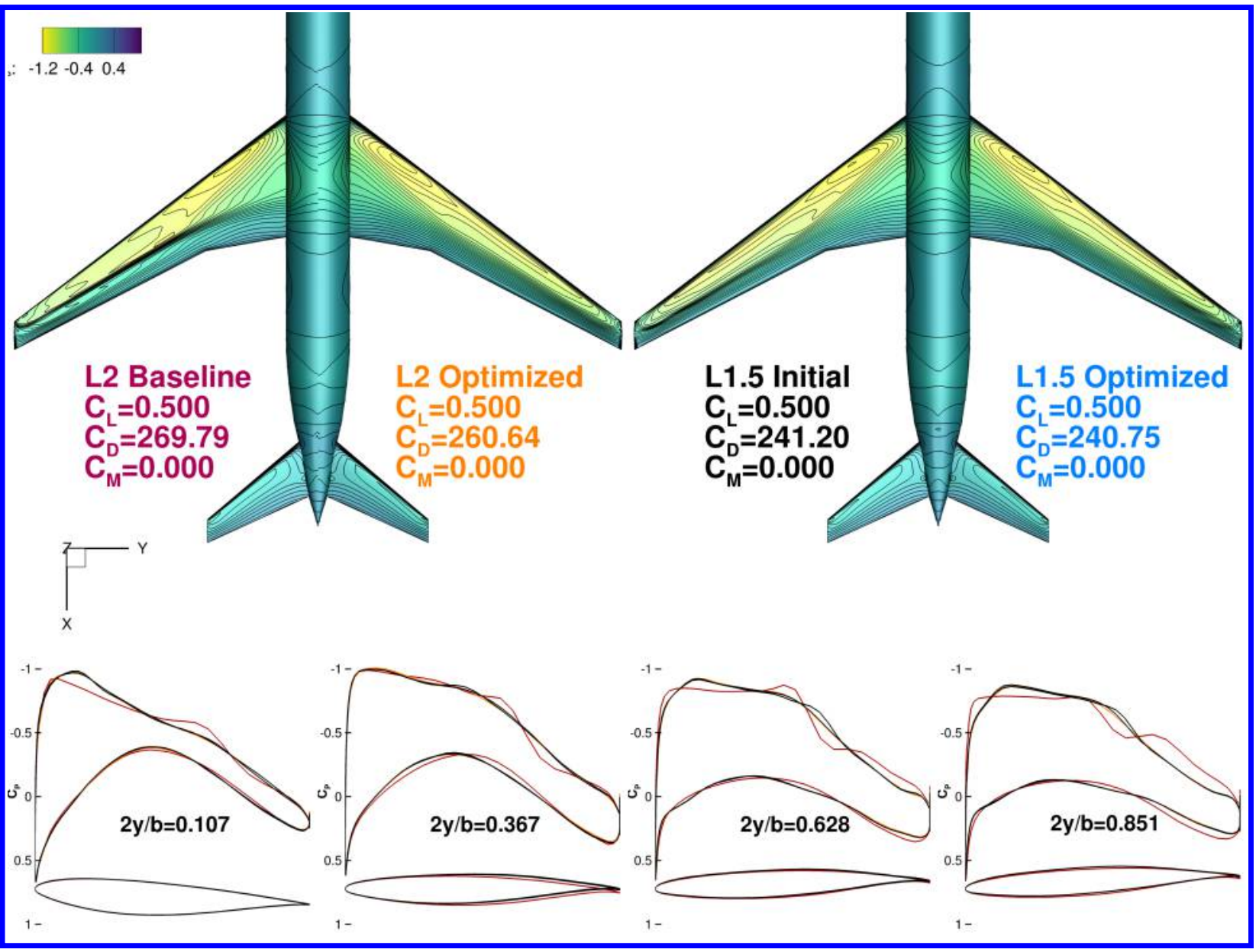

Figure 11: The coarse-grid optimum is a good starting point for the fine-grid optimization.

\section{Optimization Results}

In this section we present the main results for each of the CRM aerodynamic shape optimizations. Figure 12 shows the evolution of the SNOPT merit function and optimality. The merit function is used in the augmented Lagrangian formulation, and the optimality is a measure of how well the Karush-Kuhn-Tucker (KKT) conditions are satisfied [16].

The optimality tolerance was set to $1.0 \times 10^{-5}$, which is achieved for most optimizations. The L2 optimizations are limited to 150 iterations, while the L1.5 optimization are limited to a further 50 iterations. Case 5.7 uses a different objective function and is a maximization instead of a minimization. Generally, the finer optimizations with the L1.5 mesh was able to achieve the same convergence tolerance as the coarse L2 mesh.

Figures 13 and 14 show a summary of the key features of the two ADODG optimizations (Cases 5.1 and 5.2). The baseline configuration results are shown in red, while the optimized results are shown in blue. The planform view of the wing and fuselage shows the $C_{p}$ contours of the baseline geometry (left) and the optimized geometry (right) at the nominal operating condition $\left(M=0.85, C_{L}=0.5\right)$. Just below the planform view, the front view also shows the $C_{p}$ contours and adds a visualization of the shock surface [26]. Below this, we plot the spanwise lift, twist, and thicknessto-chord ratio $(t / c)$ distributions. A reference elliptic lift distribution is shown in gray. The right side of the figure displays the cross-sectional shapes and $C_{p}$ distributions at the five spanwise locations indicated in the planform view. Finally, the bottom right plot shows the drag divergence behavior for three lift coefficients: $C_{L}=(0.45,0.50,0.55)$.

Case 5.1 is similar to the wing-alone optimization previously presented by the authors [10]. The case is referred to as "aerodynamic shape optimization without thickness reduction" in the reference. In that case, the wing-alone configuration was optimized at the same Mach number and lift coefficient, but at much lower wind-tunnel Reynolds number of $5 \times 10^{6}$. Otherwise, the two optimization optimizations are comparable. The previous work indicated that a 10.5 drag count reduction was possible for the CRM wing-alone configuration. This compares well with the 8.6 count 

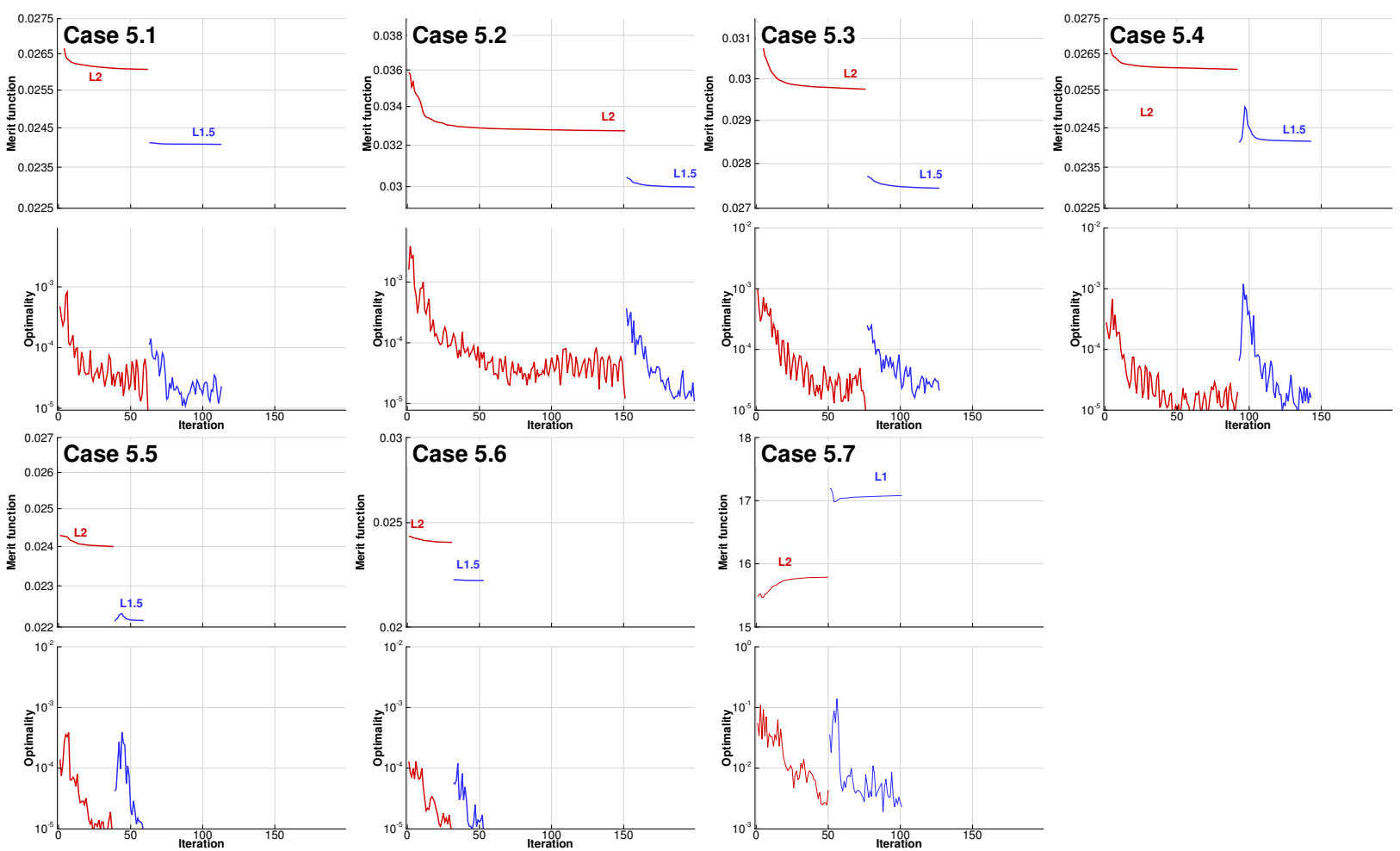

Figure 12: Merit function and optimality evolution for each optimization case.

reduction we obtain in full wing-body-tail configuration studied herein. The cross-sectional plots of the airfoils at various spanwise sections show how little the shape is modified to obtain the substantial change in performance. The drag divergence curves highlight the single-point design nature of the optimized configuration. A drag dip is present at the on-design condition, but the performance is worse at most other Mach numbers and lift coefficients.

Case 5.2 adds two additional equally-weighted operating conditions near the buffet onset boundary. Unlike Case 5.1, for which we obtain a shock-free wing, Case 5.2 results in double shocks at the nominal operating condition. In this case, the drag at the nominal operating condition actually increased by 2.8 counts. The drag divergence curves indicate a significant drag penalty across the lower Mach numbers but it does have a much higher drag divergence Mach number than the baseline design.

While drag coefficient divergence curves yield useful insights into optimized designs, it is particularly instructive to examine the 2D analogue in the form of $M L / D$ contours. In the context of transonic transport wing design, $M L / D$ is a better measure of performance, since it includes the benefit that flying faster has on the overall aircraft efficiency. This overall performance can be approximated by the Breguet range equation:

$$
R=\frac{M a}{c} \frac{L}{D} \ln \left(\frac{W_{1}}{W_{2}}\right)
$$

where $L / D$ is the lift-to-drag ratio, $a$ is the speed of sound, $c$ is the thrust-specific fuel consumption, and $W_{1}$ and $W_{2}$ are the initial and final cruise weights, respectively. For a purely aerodynamic optimization, only $L$ and $D$ vary if we assume a constant $c$ and weight ratio $W_{1} / W_{2}$, so we are left with $M L / D$.

The detailed procedure for generating contour plots is detailed in Appendix A.The contour plots are generated using the L1.5 grid, and we ignore the additional drag associated with the nacelle, pylon and vertical stabilizer. However, the estimated additional drag from these components happens to be about the same as the spurious drag for this mesh, and thus the resulting $M L / D$ values are realistic. Contour plots for all seven optimizations including the baseline design are shown in Fig. 15.

The contours in each figure extend up to the buffet onset prediction curve shown in red. The orange curve shows the buffet onset prediction using the $\Delta \alpha=0.1$ method described in Section III. There are several regions where the orange curves are missing data, which is due to the failure of finding an intersection between the two lift curves. 


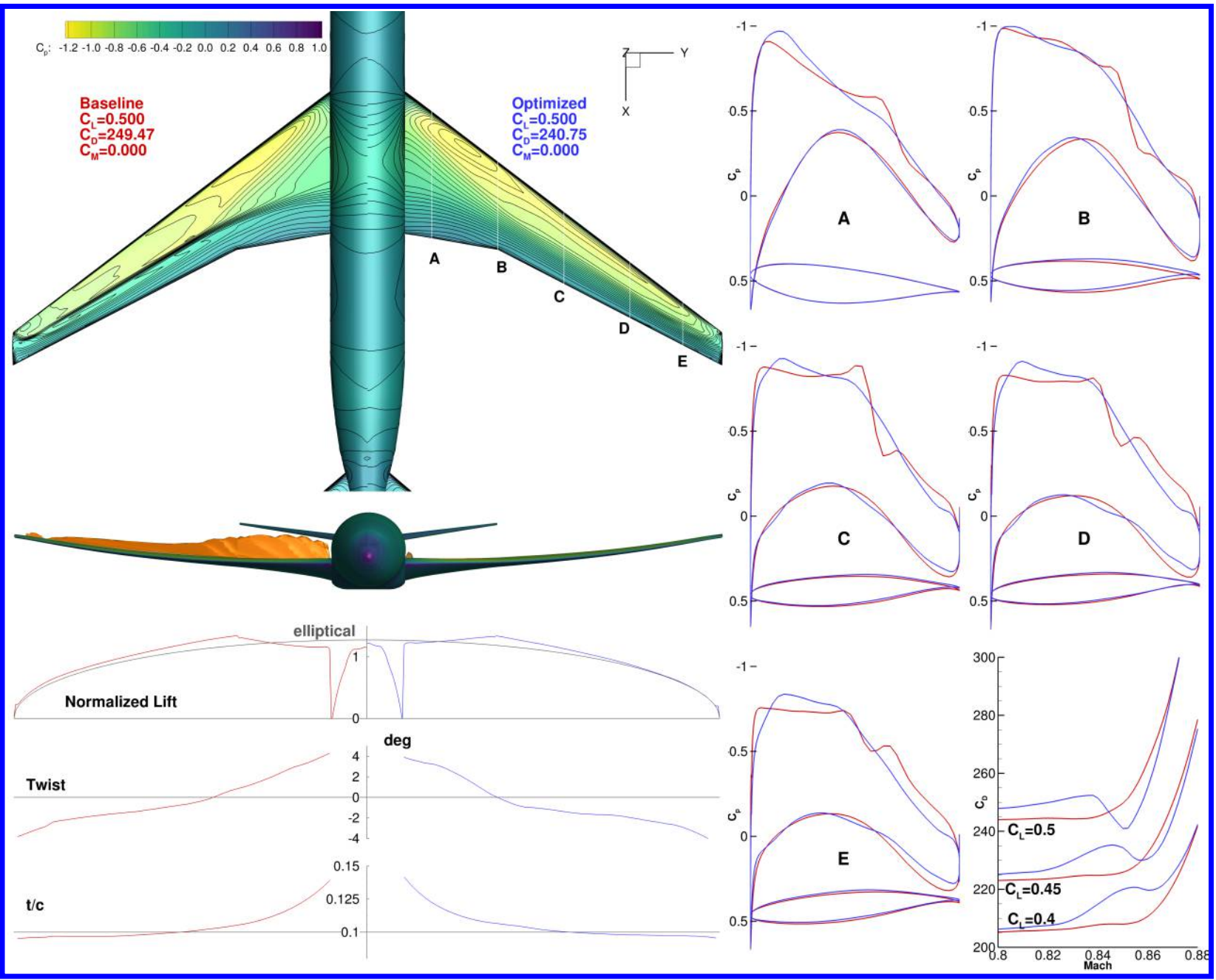

Figure 13: High performance is achieved for the single point design at the nominal operating condition.

Overall, the separation sensor method continues to compare well with the $\Delta \alpha=0.1$ method despite the large changes in the buffet onset boundary.

The blue curve represents the $1.3 \mathrm{~g}$ to buffet boundary and is computed directly from the red buffet onset curve. Only operating conditions below the $1.3 \mathrm{~g}$ curve can be considered for normal operation. The absolute maximum $M L / D$ value for each configuration is shown in pink. Two specific contours for the optimization configuration (one for the baseline configuration) are highlighted: the contour of $99 \%(M L / D)_{\max }$ for the particular design is shown in blue and the contour of $99 \%(M L / D)_{\max }$ of the baseline configuration is shown in red. The motivation for plotting these $99 \%$ contours is that airliners typically fly between the Mach number yielding maximum range (approximated by the maximum $M L / D$ value in the figures) and a higher Mach number that yields a $1 \%$ fuel-burn penalty, but also decreases in the flight time. The area enclosed by both of these contours is used to quantify the design's robustness in these figures. The areas are scaled by a factor of $100^{2}$ such that the area of the rectangle measuring 0.01 in $M$ and 0.01 in $C_{L}$ has an area of 1.0.

The design operating conditions listed in Table 3 are shown as diamonds. The operating conditions considered for the objective function are shown in black, while the buffet onset constraint conditions are shown in red.

There are two additional regions that are highlighted in black and orange. We refer to these as integration regions. They are constructed as follows: The Mach range is from 0.83 to 0.86 , which corresponds to the typical range of operating Mach numbers for an aircraft like the CRM. The upper line corresponds to the $1.3 \mathrm{~g}$ buffet boundary, which is equivalent to specifying the maximum altitude the aircraft may fly for a particular weight. The bottom line corresponds to the reduced $C_{L}$ for a $4000 \mathrm{ft}$ decrease in altitude. To put it in another way, the integration region contains all operating conditions within $4000 \mathrm{ft}$ of the buffet-constrained ceiling and at all normal operating Mach numbers. It 


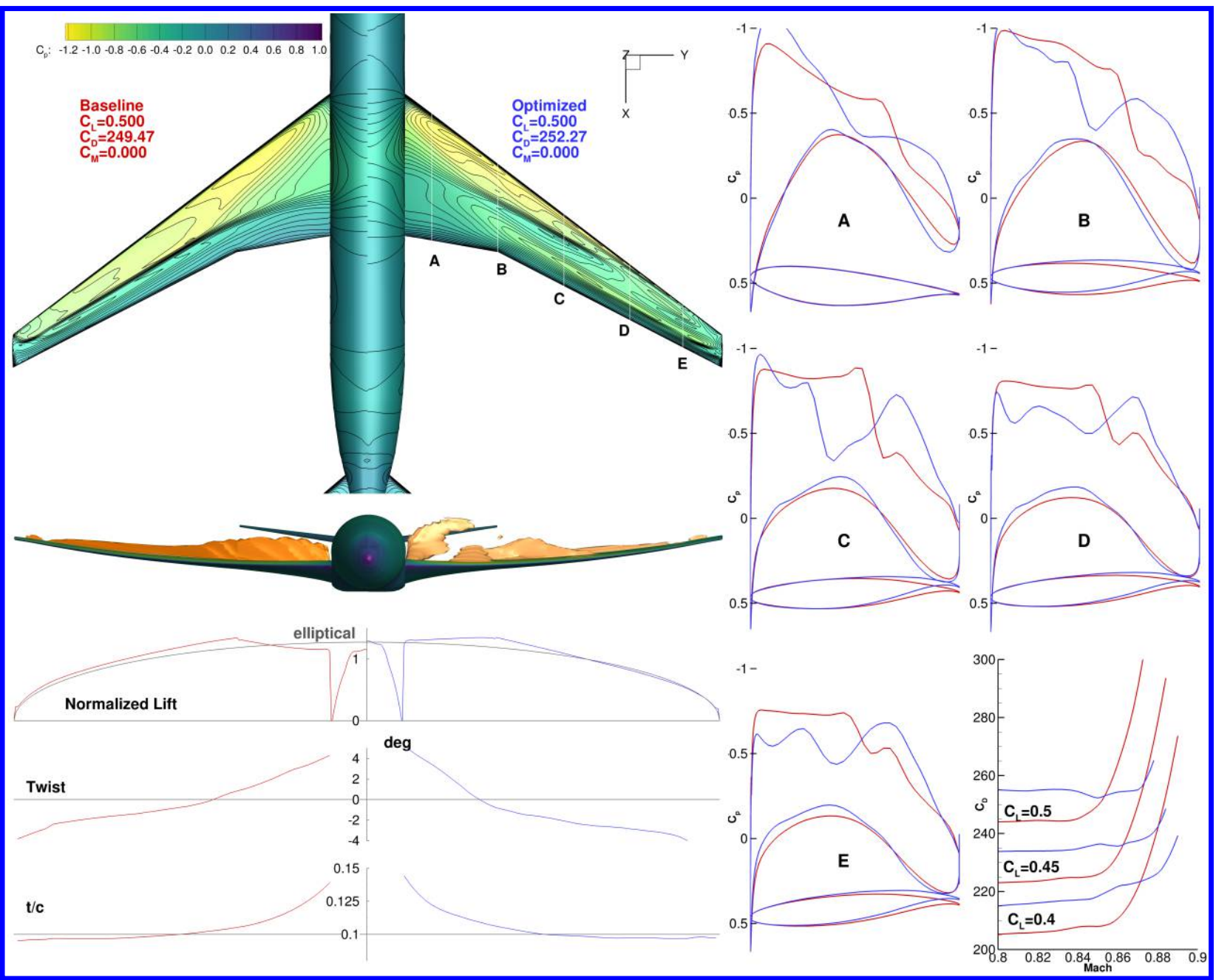

Figure 14: Performance is sacrificed across a large range of Mach numbers to obtain a small amount of improvement at the highest Mach numbers.

is likely that the aircraft spends the vast majority of cruising flight inside this region. The black integration region corresponds to the baseline design, while the orange regions are adjusted to reflected the actual $1.3 \mathrm{~g}$ buffet onset boundary for each design. Additionally, the upper edge of the black region gives an indication of how the buffet onset boundary has changed for each design relative to the baseline configuration for the specific Mach range of integration.

Figure 16 displays a different visualization of the data already shown in Fig. 15. Here, we plot the percentage change of each design relative to the baseline configuration. Note that the plot region is limited to flight conditions below the buffet boundary corresponding to each design. The color of the boundary indicates which is one active: The black boundary indicates the baseline buffet boundary is active, meaning the optimized design boundary is higher than the baseline. The orange boundary means the optimized boundary is active, and thus lower than the baseline buffet boundary. The integration region for each configuration is also shown in orange.

The contour plots give a much more complete understanding of the optimized designs. Unsurprisingly, the single point optimization-Case 5.1-was able to produce the highest $M L / D$ value, which is almost exactly matched to the design operating condition. However, without a way to constrain the buffet onset boundary, the $(M L / D)_{\max }$ is now above the $1.3 \mathrm{~g}$ boundary, which means that this high performance point cannot be achieved in practice because it falls outside the normal flight envelope. The $99 \%(M L / D)_{\max }$ contour (blue) is small, indicating a highly localized point-design. Despite the highest $M L / D$ value, the average $M L / D$ in its own integration region (orange) is $4.6 \%$ worse than the baseline design.

For Case 5.2, the addition of operating conditions at the edge of the buffet onset envelope has the effect of substantially improving the buffet boundary over the entire range of Mach numbers. This case resulted in the most robust 
buffet onset behavior of all cases. However, the $(M L / D)_{\max }$ has barely improved over the baseline design (17.18 vs. 17.13). Worse, as in Case 5.1, the high performance region lies almost entirely outside the $1.3 \mathrm{~g}$ buffet onset boundary, rendering the high performance region unattainable. Even for this case, the $M L / D$ average in the integration region is slightly worse $(-0.5 \%)$ than the baseline design. Note that the increased performance afforded by the higher buffet boundary is only possible if the baseline aircraft is buffet limited in altitude over the specific range of Mach numbers, as opposed to thrust limited. If the aircraft were thrust limited over the integration range, the obtainable performance would be the value obtained by integration of the black integration region.

In Case 5.3, we attempt to improve upon Case 5.2 by reducing the weighting factor for the near-buffet conditions. For this case, the nominal operating condition has weight of $2 / 3$, while the remaining two points have weights of $1 / 6$ each. The adjusted weightings yield a much more useful design. This is the first case where a significant portion of 99\% $(M L / D)_{\max }$ contour falls within the integration region. Additionally, the design is robust, as evidenced by the larger area enclosed by the blue contour when compared to the baseline design. As with the two previous cases, the increased performance is only possible if the aircraft is able to operate at higher altitudes. The other issue with this case is that the specific weightings were arbitrarily picked. These particular weights yielded acceptable results, but there is no guarantee these weight values would work well for another configuration or optimization problem.

Case 5.4 is the first optimization to use the separation sensor directly as an optimization constraint. Case 5.4 retains the same operating conditions as Cases 5.2 and 5.3, but instead of having the drag from the flight conditions near the buffet boundary contribute to the drag objective function, it uses the buffet onset flight conditions to compute the separation sensor and constrain its value. Note that there is a slight discrepancy between the operating conditions (red diamonds) and the buffet onset boundary itself. The reason is that the buffet onset conditions are analyzed using the JST scalar dissipation scheme, which results in a solution with more dissipation. The scalar scheme provides the increased robustness necessary for optimization, which is unnecessary for the contour plot evaluations. The more dissipative scalar scheme slightly under-predicts the area of separated flow, and thus the buffet boundary is lower when analyzed with the matrix scheme for the contour plot. Overall, the performance of this design is similar to that of the single-point optimization (Case 5.1). Most of the high performance region lies outside the integration region. However, the performance reduction is not as pronounced as with Case 5.1, with a $1.6 \%$ performance reduction in the original integration region and almost zero for the on-design integration region. However, there is a small improvement in the buffet onset boundary.

After analyzing the results from Cases 5.1-5.4, we noticed that the nominal design point was always located towards the upper side or even completely outside the integration region. It is perhaps no surprise that all optimization discussed thus far failed to improve the performance in the baseline integration region. To address this issue, we formulated a multipoint optimization, Case 5.5. Previous optimizations performed by the authors on the CRM wing along configuration have shown that five operating conditions arranged as a cross in $M-C_{L}$ space results is highly robust designs [25]. Since our goal is to improve the performance in the original integration region, we distribute five operating conditions as follows. The nominal Mach number is reduced to 0.845 for the first three operating conditions. The first point lies on the $1.3 \mathrm{~g}$ buffet onset boundary, while the second two points are at $C_{L}$ values corresponding to 2000 and $4000 \mathrm{ft}$ below. The two remaining points are $2000 \mathrm{ft}$ lower than the buffet onset boundary at \pm 0.01 Mach number. The buffet onset conditions are taken at $M=0.85, C_{L}=0.65$ and $M=0.89, C_{L}=0.41$. The later point is taken from the baseline design buffet onset boundary. The overall performance of this case is superior to all previously discussed cases. The performance in the baseline integration region has now increased by $1.2 \%$, the performance of the updated integration region increase by $2.0 \%$. The design is very robust, as shown by area inside the $99 \%(M L / D)_{\max }$ contour. Further, the maximum performance point is located inside the operating envelope.

Next we developed Case 5.6, which is designed to investigate the effect of removing the buffet onset conditions present in Case 5.5. We wish to answer the question: Is a multipoint optimization near the design operating condition sufficient to ensure a robust buffet onset envelope? Unsurprisingly, without the buffet onset constraints, the $1.3 \mathrm{~g}$ onset boundary dropped slightly over the integration envelope, pushing the integration region into a lower performance region. The average $M L / D$ for the integration region is 16.88 , only $0.6 \%$ higher than the baseline configuration. This is much smaller than the $2.0 \%$ improvement obtained in Case 5.5.

For the last case, Case 5.7, we formulate a different design optimization problem. We wish to remove the requirement of specifying "design" lift coefficients and let the optimization itself determine the ideal on-design condition. All cases presented thus far are lift-constrained drag minimizations with fixed operating conditions. The fixed operating conditions also include fixing the $C_{L}$ for the buffet onset locations. In the formulation of Case 5.7, we want the optimization to adjust the single nominal operating condition directly. The remaining operating conditions are then explicitly linked to this design $C_{L}$. More specifically, the high $C_{L}$ buffet onset conditions must have 1.35 times the lift of the nominal cruise Mach. The high Mach buffet case must have the same physical lift as the nominal operating condition at $M=0.89$. Finally, the remaining operating conditions move vertically in sync with the changing design 
$C_{L}$. The modified optimization formulation is as follows:

$\begin{array}{rll}\text { maximize } & \sum_{i=1}^{N} \mathcal{W}_{i} M_{i} L_{i} / D_{i} & \text { Quantity } \\ \text { with respect to } & \text { Wing cross sectional shape } & 240 \\ & \text { Wing twist } & 9 \\ & \text { Angle of attack }\left(\alpha_{i}\right) & \mathrm{N} \\ & \text { Tail rotation angle }\left(\eta_{i}\right) & \mathrm{N} \\ & \text { Design } C_{L} & 1 \\ \text { subject to } & C_{L_{i}}-C_{L_{i}}^{*}=0.0 & \mathrm{~N} \\ & C_{M_{y_{i}}}=0.0 & \mathrm{~N} \\ & t_{j} \geq t_{\text {CRM }_{\text {CR }}} & 750 \\ & \operatorname{Sep}_{i} \leq 0.04 & \mathrm{~N}\end{array}$

Note that operating conditions (diamonds) shown in Fig. 15 are the optimized values. The optimization increased the nominal design $C_{L}$ from the initial value of 0.490 (the same value as used in Cases 5.5 and 5.6) and increased it to 0.520 , an increase of 0.03 in $C_{L}$. This increase is only possible due to a corresponding increase in the buffet onset boundary. The previous optimizations, especially Case 5.2, showed that there can be a significant penalty in cruise drag for a higher buffet boundary. For Case 5.7, we have given the optimizer sufficient information to make this trade-off optimal. This results in a slightly higher average performance than Case 5.5 (17.13 vs. 17.11), as well as a higher buffet onset boundary. The design is also highly robust, exhibiting the largest $99 \%(M L / D)_{\max }$ contour of all cases.

Further insight into the differences between the optimized designs can be inferred from Fig. 16. It is particularly interesting to see that there is a region between $M=0.86$ and $M=0.88$ at low $C_{L}$ that is universally worse on all optimized designs. This is particularly noticeable on the single-point type designs (Cases 5.1 and 5.4). This effect is the least evident in Case 5.5, where there is an improvement over almost the entire contour region. Compared to Case 5.7, the higher buffet onset performance appears to be correlated with the reduced low $C_{L}$ performance. The performance reduction at lower lift coefficients in Case 5.7 is limited to less than $2 \%$, which is acceptable given the performance increase at the higher lift coefficients.

\section{Grid Convergence}

We perform grid convergence studies for the baseline geometry as well as all the optimized configurations. For the grid convergence study, we take the optimized geometry from the L1.5 mesh and apply it to the each of the four meshes in sequence. The drag convergence for each mesh configuration is shown in Fig. 17a. The drag coefficient, when plotted against the grid factor $\left(N_{\text {cells }}^{-2 / 3}\right)$ is approximately linear, indicating second order convergence. However, the finest analyzed mesh (L0.5) does not fall directly on the line, indicating a more highly resolved mesh is necessary to determine a grid-converged value. However, for aerodynamic shape optimization, we are generally more concerned about the change in drag coefficient resulting from a design change as opposed to the grid converged value. Figure 17b shows the change in drag coefficient for each configuration on each mesh level. There is remarkably little variation across each mesh level. This can be attributed to the fact that the spurious drag remains roughly constant for a given mesh, independent of the design modifications. The maximum variation between the improvement on the L1.5 meshthe finest mesh for which optimization was performed - and the L0.5 mesh is 0.63 counts (in Case 5.6). Given the much larger computational cost of performing optimization with the L1 or L0.5 meshes, and the small difference in predicted drag improvement when using these finer meshes, using the L1.5 mesh for our optimizations is a good choice.

\section{Computational Cost}

Multipoint three-dimensional RANS-based aerodynamic shape optimizations are costly from a computational perspective, and we make every effort to reduce the total cost of the optimizations. Table 4 lists the total CPU cost, in processor hours, required to generate the results presented in this paper. All the computations were performed on nodes with two 4-core E5540 CPUs running at $2.53 \mathrm{GHz}$ with $16 \mathrm{~GB}$ of RAM per node. The nodes are connected with QDR InfiniBand. All L2 and L1.5 meshes were run on 64 cores, while the L1 and L0.5 meshes for the grid convergence study used 128 cores.

Approximately $63 \%$ of the total computational time was expended in the optimizations, and the remainder of the time was used for post-processing. The $M L / D$ contour plots are particularly costly, since each plot requires approximately 400-500 individual CFD evaluations. 
Table 4: Computational cost breakdown in CPU hours.

\begin{tabular}{lrrrrr}
\hline Case & L2 optimization & L1.5 optimization & Contour & Grid convergence & Total \\
\hline Baseline & - & - & 1346 & 817 & 2162 \\
5.1 & 289 & 611 & 1270 & 1009 & 3179 \\
5.2 & 2378 & 2394 & 1795 & 1121 & 7688 \\
5.3 & 1290 & 2505 & 1750 & 910 & 6457 \\
5.4 & 1507 & 2602 & 1384 & 1024 & 6518 \\
5.5 & 2,090 & 3506 & 1392 & 830 & 7369 \\
5.6 & 1,111 & 1803 & 1147 & 610 & 4673 \\
5.7 & 4,136 & 6623 & 1800 & 696 & 13255 \\
\hline Total & 12802 & 19567 & 11886 & 7019 & 51303 \\
\hline
\end{tabular}

\section{Conclusions}

A new formulation for the prediction of buffet onset is presented. The method uses the integration of a separation sensor along with a cut-off value to estimate when buffet first occurs, and can be directly evaluated of with only one CFD solution. The method is shown to compare well with the $\Delta \alpha=0.1$ method for the CRM configuration, as well as for various optimized designs. A comparison with root bending moment data obtained from wind tunnel experiments also shows good predictive capabilities. The separation sensor method is particularly well suited for formulating a constraint in gradient-based optimization, as it is easy to implement in a discrete adjoint optimization framework, and the resulting function (although highly nonlinear) is smooth.

To demonstrate the effectiveness of the propose approach, we presented a series of seven optimizations of the CRM wing-body-tail configuration. All optimizations are performed with respect to 216 shape variables, 9 twist variables, and tail rotation angle subject to lift, pitching moment, volume, thickness, and separation constraints. A two-level sequential optimization approach is employed to reduce the overall computational cost required.

At the nominal operating condition of $M=0.85, C_{L}=0.5$, the single-point optimization (Case 5.1) reduced the drag coefficient from 249.5 counts to 240.9 counts, a reduction of $3.4 \%$. For a more complete comparison between the optimized designs, we plotted contours of $M L / D$ in the $M-C_{L}$ space. This is a highly visual and intuitive way of comparing the performance and robustness of optimized configurations.

In Case 5.2, we added two operating conditions near buffet onset conditions. The performance at these points increased substantially, and this design produced a high buffet onset boundary. However, the overall performance as measured by a typical operating envelope was lower than the baseline. Weighting the nominal cruise point (Case 5.3) more than the off-design condition improves the performance, but requires knowledge of how to choose the appropriate weights, which may be case dependent.

In Case 5.4, we introduced the use of the separation sensor for controlling the buffet onset boundary directly. While this is effective, the overall performance of the optimized design was lacking, and the performance was lower than the weighted points approach in Case 5.3.

The remaining cases used five main operating conditions to produce more robust designs. Case 5.5 showed performance improvement over nearly the entire transonic range, while simultaneously improving part of the buffet onset boundary. Case 5.6 removed the buffet onset conditions, demonstrating that a multipoint optimization with all conditions near the on-design condition is not sufficient, as a the buffet onset boundary encroached in the cruise performance region, reducing the average usable improvement from $2.0 \%$ for Case 5.5 to only $0.6 \%$. Finally, in Case 5.7, we performed an $M L / D$ maximization with automatic determination of the operating $C_{L}$, which improved the average performance over Case 5.5, while pushing the buffet onset boundary beyond the operational envelope.

\section{Acknowledgments}

Funding for this research was provided in part by NASA under grant number NNX11AI19A. 


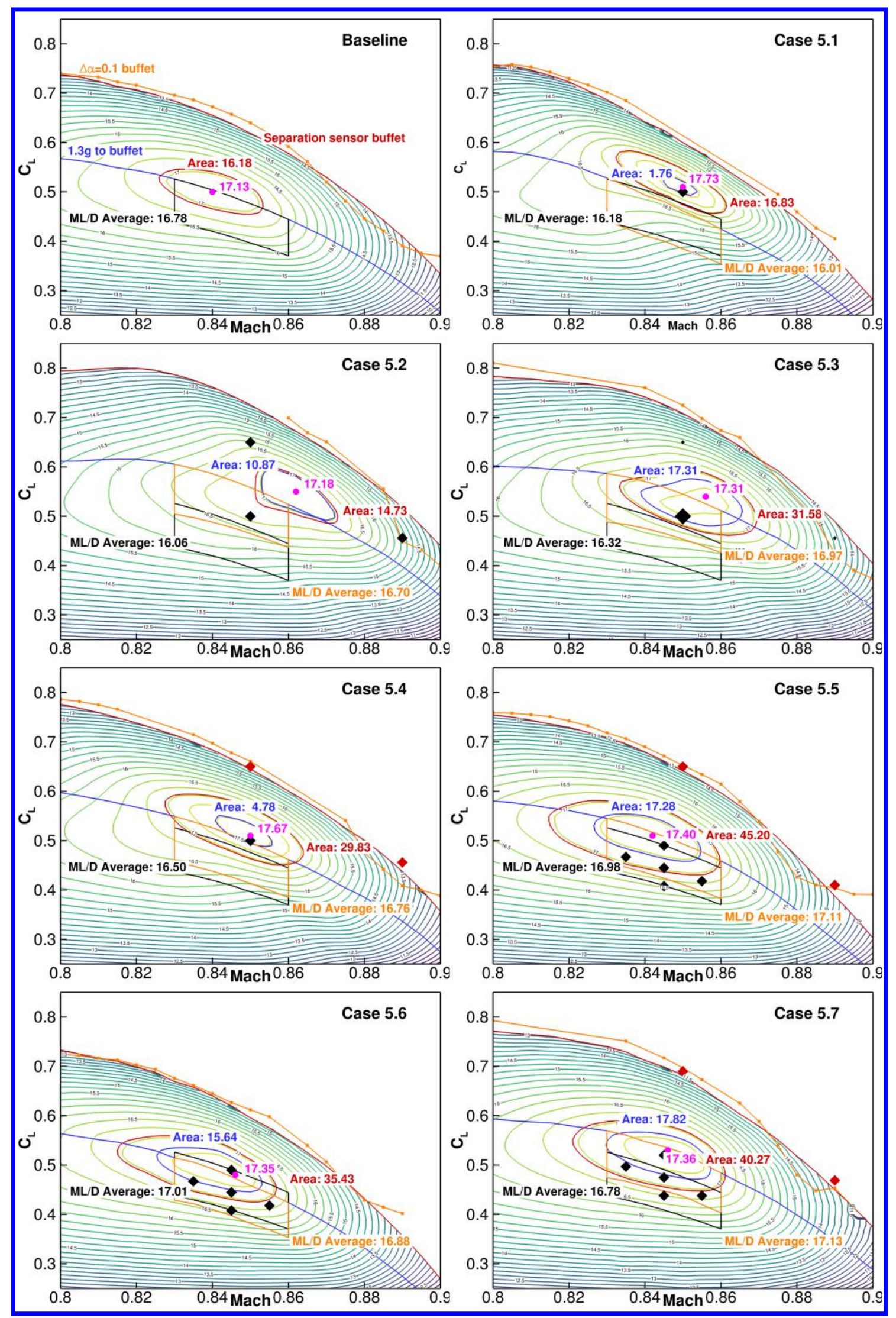

Figure 15: Contours of $M L / D$ for the baseline and each optimized configuration. 


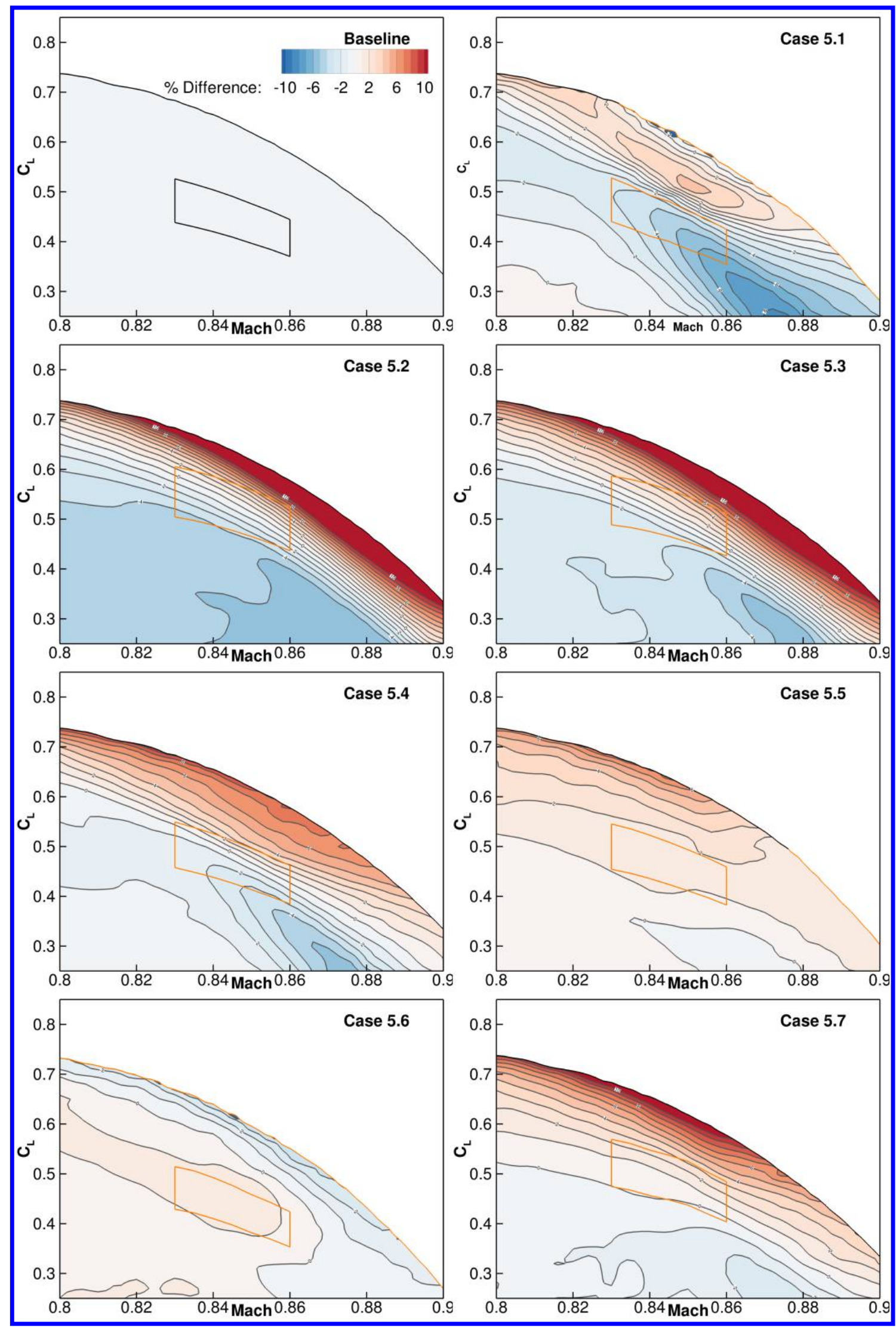

Figure 16: Percent difference in $M L / D$ between the baseline design and the optimized configuration. 


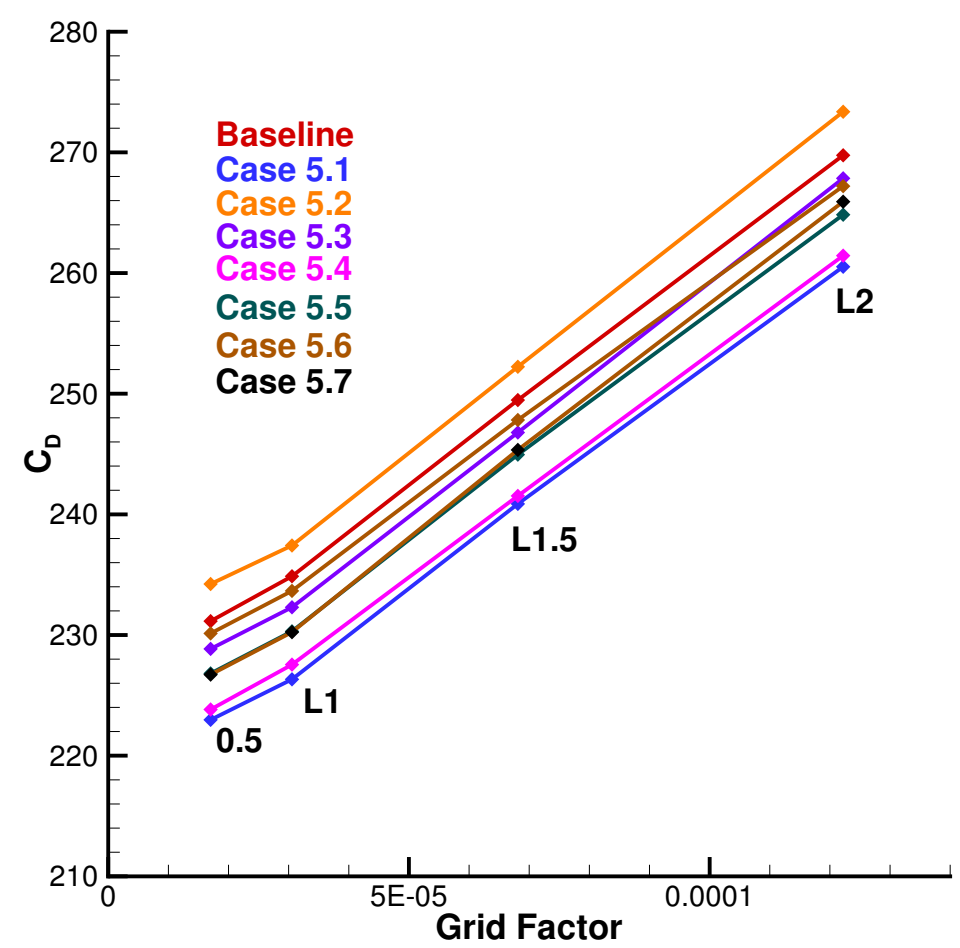

(a) The change in drag is constant between grid levels

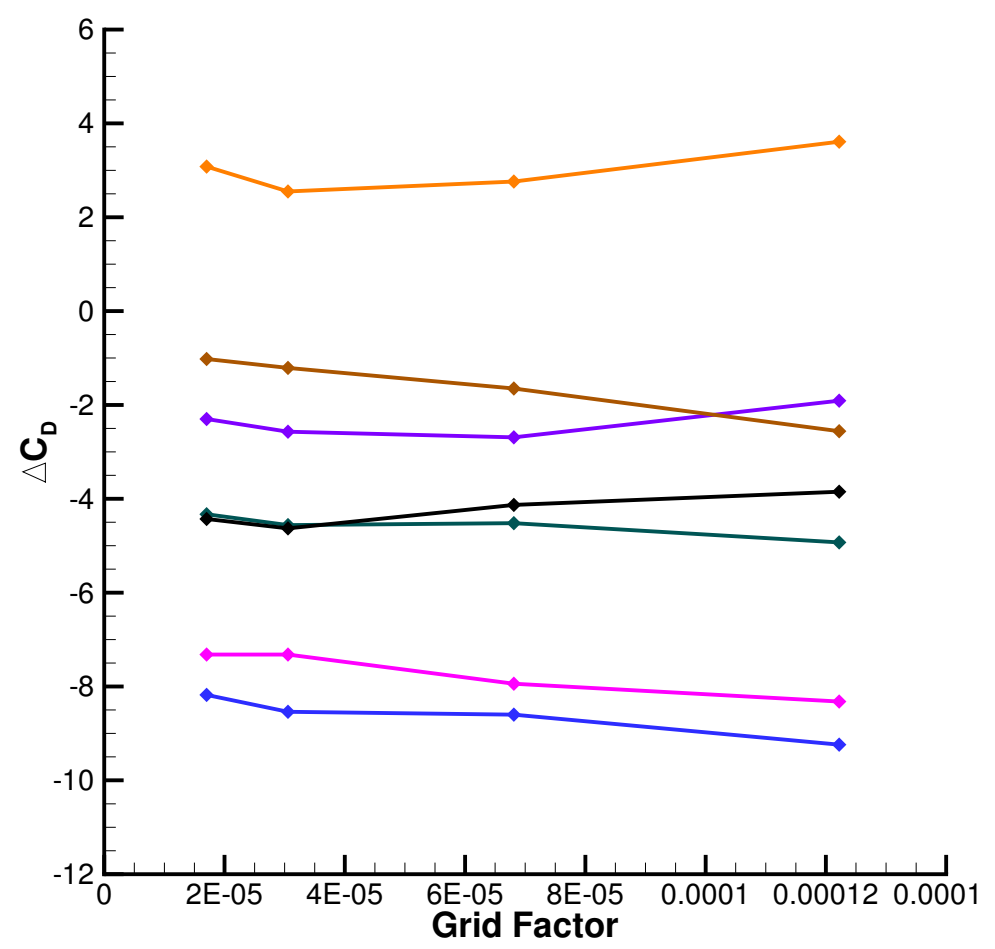

(b) The change in drag is roughly constant for all designs between grid levels.

Figure 17: Grid convergence study for baseline and all optimized configurations. 


\section{References}

- [1] Kenway, G. K. W., Kennedy, G. J., and Martins, J. R. R. A., "Scalable Parallel Approach for High-Fidelity Steady-State Aeroelastic Analysis and Derivative Computations," AIAA Journal, Vol. 52, No. 5, May 2014, pp. 935-951. doi:10.2514/1.J052255.

-[2] Kenway, G. K. W. and Martins, J. R. R. A., "Multipoint High-Fidelity Aerostructural Optimization of a Transport Aircraft Configuration," Journal of Aircraft, Vol. 51, No. 1, January 2014, pp. 144-160. doi:10.2514/1.C032150.

[3] van der Weide, E., Kalitzin, G., Schluter, J., and Alonso, J. J., "Unsteady Turbomachinery Computations Using Massively Parallel Platforms," Proceedings of the 44th AIAA Aerospace Sciences Meeting and Exhibit, Reno, NV, 2006, AIAA 20060421.

-[4] Mader, C. A., Martins, J. R. R. A., Alonso, J. J., and van der Weide, E., “ADjoint: An Approach for the Rapid Development of Discrete Adjoint Solvers," AIAA Journal, Vol. 46, No. 4, April 2008, pp. 863-873. doi:10.2514/1.29123.

- [5] Spalart, P. and Allmaras, S., "A One-Equation Turbulence Model for Aerodynamic Flows," 30th Aerospace Sciences Meeting and Exhibit, 1992. doi:10.2514/6.1992-439.

-[6] Lyu, Z., Kenway, G. K., Paige, C., and Martins, J. R. R. A., "Automatic Differentiation Adjoint of the Reynolds-Averaged Navier-Stokes Equations with a Turbulence Model," 21st AIAA Computational Fluid Dynamics Conference, San Diego, CA, Jul. 2013. doi:10.2514/6.2013-2581.

-[7] Mader, C. A. and Martins, J. R. R. A., "Stability-Constrained Aerodynamic Shape Optimization of Flying Wings," Journal of Aircraft, Vol. 50, No. 5, September 2013, pp. 1431-1449. doi:10.2514/1.C031956.

- [8] Mader, C. A. and Martins, J. R. R. A., "Computing Stability Derivatives and their Gradients for Aerodynamic Shape Optimization,” AIAA Journal, Vol. 52, No. 11, November 2014, pp. 2533-2546. doi:10.2514/1.J052922.

-[9] Lyu, Z. and Martins, J. R. R. A., “Aerodynamic Design Optimization Studies of a Blended-Wing-Body Aircraft," Journal of Aircraft, Vol. 51, No. 5, September 2014, pp. 1604-1617. doi:10.2514/1.C032491.

[10] Lyu, Z., Kenway, G. K., and Martins, J. R. R. A., "Aerodynamic Shape Optimization Studies on the Common Research Model Wing Benchmark,” AIAA Journal, Vol. 53, No. 4, April 2015, pp. 968-985. doi:10.2514/1.J053318.

[11] Chen, S., Lyu, Z., Kenway, G. K. W., and Martins, J. R. R. A., "Aerodynamic Shape Optimization of the Common Research Model Wing-Body-Tail Configuration," Proceedings of the AIAA Science and Technology Forum and Exposition (SciTech), Kissimmee, FL, January 2015. doi:10.2514/6.2015-1718, AIAA 2015-1718.

-[12] Kenway, G. K. W. and Martins, J. R. R. A., "Multipoint Aerodynamic Shape Optimization Investigations of the Common Research Model Wing," Proceedings of the AIAA Science and Technology Forum and Exposition (SciTech), Kissimmee, FL, January 2015. doi:10.2514/6.2015-0264.

[13] Liem, R., Kenway, G. K. W., and Martins, J. R. R. A., "Multimission Aircraft Fuel Burn Minimization via Multipoint Aerostructural Optimization,” AIAA Journal, Vol. 53, No. 1, January 2015, pp. 104-122. doi:10.2514/1.J052940.

[14] Kenway, G. K. W., Kennedy, G. J., and Martins, J. R. R. A., "Aerostructural Optimization of the Common Research Model Configuration," 15th AIAA/ISSMO Multidisciplinary Analysis and Optimization Conference, Atlanta, GA, June 2014, AIAA 2014-3274.

-[15] Luke, E., Collins, E., and Blades, E., "A Fast Mesh Deformation Method Using Explicit Interpolation,” J. Comput. Phys., Vol. 231, No. 2, Jan. 2012, pp. 586-601. doi:10.1016/j.jcp.2011.09.021.

- [16] Gill, P. E., Murray, W., and Saunders, M. A., "SNOPT: An SQP algorithm for large-scale constrained optimization," SIAM Journal of Optimization, Vol. 12, No. 4, 2002, pp. 979-1006. doi:10.1137/S1052623499350013.

- [17] Perez, R. E., Jansen, P. W., and Martins, J. R. R. A., "pyOpt: A Python-Based Object-Oriented Framework for Nonlinear Constrained Optimization," Structural and Multidisciplinary Optimization, Vol. 45, No. 1, January 2012, pp. 101-118. doi:10.1007/s00158-011-0666-3.

- [18] Lyu, Z. and Martins, J. R. R. A., “Aerodynamic Shape Optimization of an Adaptive Morphing Trailing Edge Wing," Journal of Aircraft, Vol. 52, No. 6, November 2015, pp. 1951-1970. doi:10.2514/1.C033116.

[19] Obert, E., Aerodynamic Design of Transport Aircraft, IOS Press BV, 2009.

-[20] Lovely, D. and Haimes, R., "Shock detection from computational fluid dynamics results," Proceedings of the 14th Computational Fluid Dynamics Conference, Norfolk, VA, January 1999. doi:doi:10.2514/6.1999-3285.

[21] Vassberg, J. C., DeHaan, M. A., Rivers, S. M., and Wahls, R. A., "Development of a Common Research Model for Applied CFD Validation Studies," 2008, AIAA 2008-6919.

-[22] Balakrishna, S. and Acheson, M., "Analysis of NASA Common Research Model Dynamic Data," 49th Aersopace Sciences Meeting Including the New Horizons Forum and Aerospace Exposition, 2011. doi:10.2514/6.2011-1127.

[23] Kenway, G. K. W. and Martins, J. R. R. A., "AIAA ADODG Case 5: CRM Wing-Body-Tail Optimization at Flight Reynolds Number," Tech. rep., AIAA, May 2015. 
[24] Vassberg, J. C., Tinoco, E. N., Mani, M., Rider, B., Zickuhr, T., Levy, D. W., Brodersen, O. P., Eisfeld, B., Crippa, S., Wahls, R. A., Morrison, J. H., Mavriplis, D. J., and Murayama, M., "Summary of the Fourth AIAA Computational Fluid Dynamics Drag Prediction Workshop,” Journal of Aircraft, Vol. 51, No. 4, jul 2014, pp. 1070-1089. doi:10.2514/1.c032418.

[25] Kenway, G. K. W. and Martins, J. R. R. A., "Multipoint Aerodynamic Shape Optimization Investigations of the Common Research Model Wing," AIAA Journal, 2016. doi:10.2514/1.J054154, (In press).

[26] Haimes, R., "Automated Feature Extraction from Transient CFD Simulations," Proceeding of the 7th Annual Conference of the CFD Society of Canada, Halifax, NS, May 1999.

-[27] Akima, H., "A new method of interpolation and smooth curve fitting based on local procedures," Journal of the ACM, Vol. 4, No. 17, 1970, pp. 589-602.

\section{A. Contour Plot Generation}

The generation of the contour plots shown in Fig. 15 warrants further explanation. These contours are not simple $\alpha$ sweeps, as it is necessary to determine the tail angle that gives $C_{M}=0$ at each point. Once flight condition in the contour is trimmed, the trim drag penalty is included in the computed drag coefficient. Naively performing a secant search to determine the tail angle at each point would require at least three CFD solutions. Since each contour plot requires approximately 400 trim-converged solutions, we seek an alternative approach to reduce the computational cost as much as possible. One way to achieve this is to reuse previously evaluated points to continually update the $2 \times 2$ Jacobian of the residual, $F=\left[C_{L}-C_{L}^{*}, C_{M}\right]$, with respect to $[\alpha, \eta]$, where $\alpha$ is the angle of attack, and $\eta$ is the tail rotation angle. With an accurate Jacobian, we can use Newton's method to simultaneous determine the new $\alpha$ and $\eta$ required to produce a trimmed solution at a new $C_{L}$. The full procedure is listed in Algorithm 2. An auxiliary function for computing the residual for a given $(\alpha, \eta)$ is given in Algorithm 1. In practice, only one sub iteration is necessary for most points, since the $C_{L}$ and $C_{M}$ functions are not highly nonlinear functions of $\alpha$ and $\eta$ over most of contour region. Generally, additional sub-iterations are only necessary as buffet is approached, due to the more rapid variation in the lift curve slope. For example, the contour for the baseline configuration required 430 function evaluations to produce 356 converged trim- $C_{L}$ solutions, an increase of only $20 \%$. Note that we only check for the convergence of $C_{M}$, since it is not critical that the lift coefficients precisely match the specified target. Once all the raw data is generated, $C_{L}, C_{D}, C_{M}$ and separation sensor values are interpolated using an Akima spline [27] to produce a regular $M-C_{L}$ grid. This regular grid is then used for further computations, such as the difference plots shown in Fig. 16, the drag divergence curves in Figs. 13 and 14, as well as the for the extraction of particular contours and the computation of the average performance over specific integration regions.

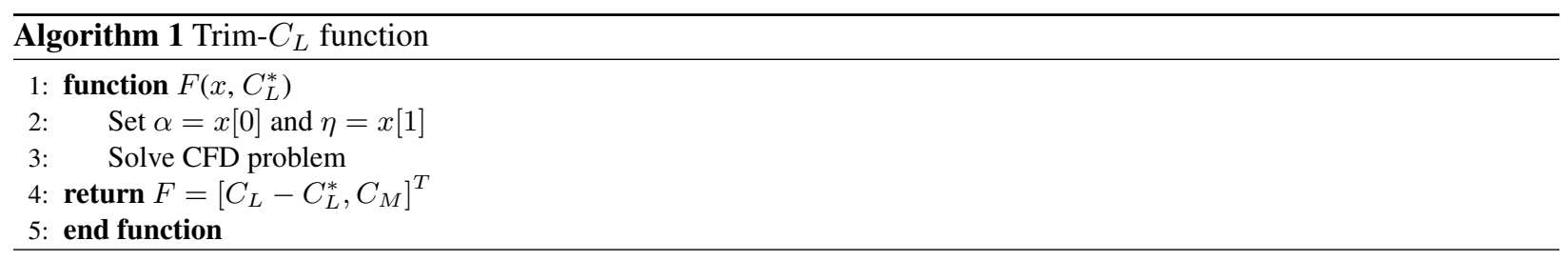




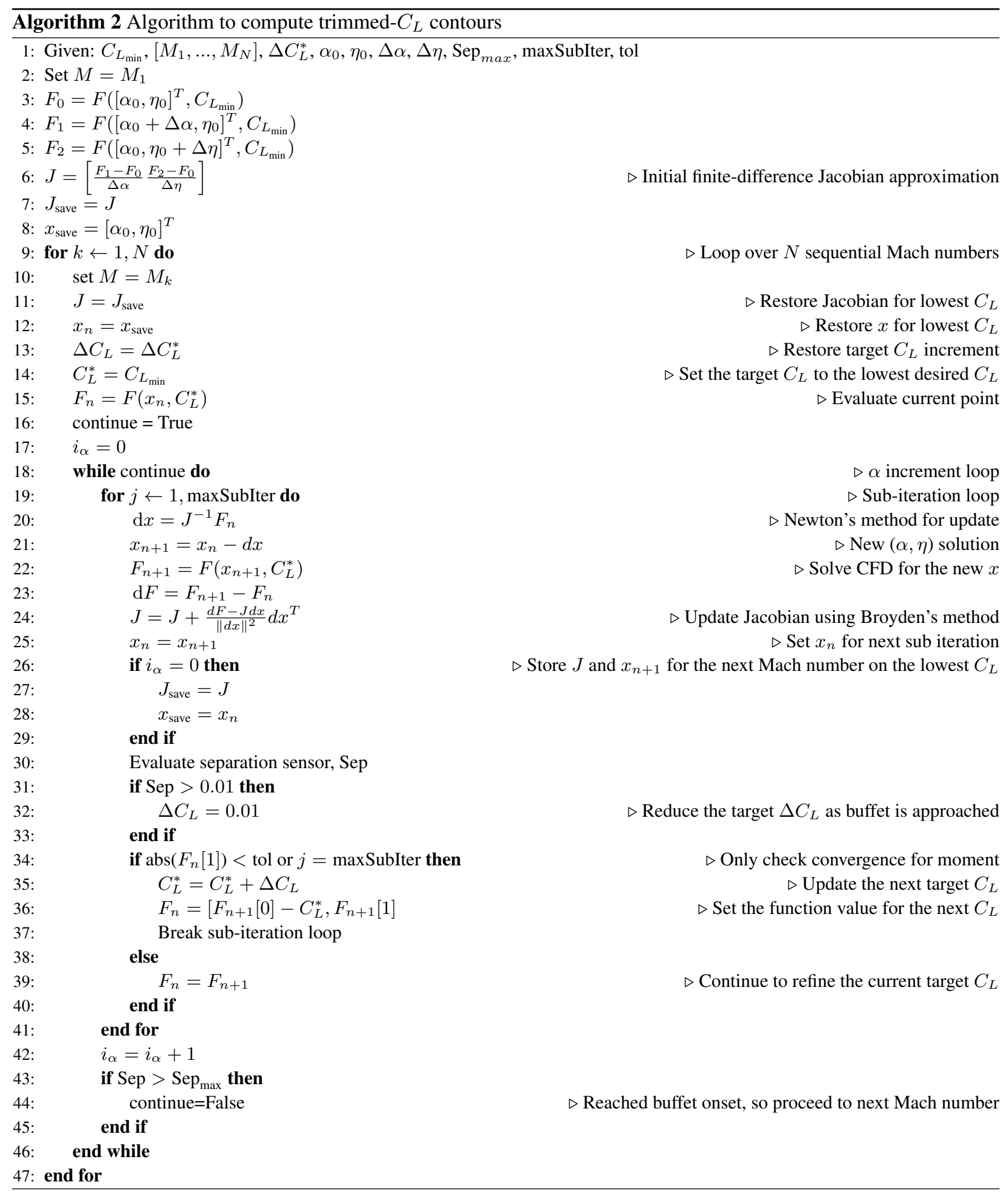


This article has been cited by:

1. Shenren Xu, Sebastian Timme, Orest Mykhaskiv, Jens-Dominik M?ller. 2017. Wing-body junction optimisation with CADbased parametrisation including a moving intersection. Aerospace Science and Technology . [Crossref]

2. Gaetan K. Kenway, Asitav Mishra, Ney R. Secco, Karthikeyan Duraisamy, Joaquim Martins. An Efficient Parallel Overset Method for Aerodynamic Shape Optimization . [Citation] [PDF] [PDF Plus]

3. Christopher Lee, David Koo, David W. Zingg. 2017. Comparison of B-Spline Surface and Free-Form Deformation Geometry Control for Aerodynamic Optimization. AIAA Journal 55:1, 228-240. [Abstract] [Full Text] [PDF] [PDF Plus] 